\title{
Fractional N-Laplacian boundary value problems with jumping nonlinearities in the fractional Orlicz-Sobolev spaces
}

\author{
Q-Heung Choi ${ }^{1}$ and Tacksun Jung ${ }^{2^{*}}$ (D)
}

\section{"Correspondence:}

tsjung@kunsan.ac.kr

${ }^{2}$ Department of Mathematics, Kunsan National University, Kunsan, 573-701, Korea

Full list of author information is

available at the end of the article

\section{Springer}

\begin{abstract}
We investigate the multiplicity of solutions for problems involving the fractional $\mathrm{N}$-Laplacian. We obtain three theorems depending on the source terms in which the nonlinearities cross some eigenvalues. We obtain these results by direct computations with the eigenvalues and the corresponding eigenfunctions for the fractional N-Laplacian eigenvalue problem in the fractional Orlicz-Sobolev spaces, the contraction mapping principle on the fractional Orlicz-Sobolev spaces and Leray-Schauder degree theory.

MSC: $35 \mathrm{~A} 01 ; 35 \mathrm{~A} 16 ; 35 \mathrm{~J} 60$

Keywords: Fractional N-Laplacian operator; Fractional Orlicz-Sobolev space; Fractional N-Laplacian eigenvalue problem; Jumping nonlinearity; Contraction mapping principle; Leray-Schauder degree theory
\end{abstract}

\section{Introduction}

In this paper we consider the existence and multiplicity of solutions in $W_{0}^{s} L_{G}(\Omega) \cap C(\Omega)$ for the following fractional N-Laplacian Dirichlet boundary value problems with jumping nonlinearties;

$$
\begin{aligned}
& \begin{aligned}
(-\Delta)_{g}^{s} u(x)= & b^{(s, g, \alpha)} g(|u(x)|) \frac{u(x)^{+}}{|u(x)|}-a^{(s, g, \alpha)} g(|u(x)|) \frac{u(x)^{-}}{|u(x)|} \\
& +\tau g\left(\left|\phi_{1}^{(s, g, \alpha)}(x)\right|\right) \frac{\phi_{1}^{(s, g, \alpha)}(x)}{\left|\phi_{1}^{(s, g, \alpha)}(x)\right|}
\end{aligned} \\
& \int_{\Omega} G(|u(x)|) d x=\alpha, \quad \text { in } \Omega, \\
& u(x)=0 \quad \text { on } \partial \Omega,
\end{aligned}
$$

where $\Omega$ is a bounded domain of $\mathrm{R}^{N}$ with a smooth boundary $\partial \Omega, N \geq 1,0<s<1, \alpha>0$, $g$ is an odd, strictly increasing continuous function from $[0, \infty)$ onto $[0, \infty)$ with $g(0)=0$ and $\lim _{t \rightarrow \infty} g(t)=\infty, G(t)=\int_{0}^{|t|} g(\tau) d \tau, t \in R$, is a N-function, $(-\Delta)_{g}^{s} u(x)$ is the nonhomogeneous fractional N-Laplacian operators defined as: for each $x \in \Omega$ and any $u$ in the

(c) The Author(s) 2021. This article is licensed under a Creative Commons Attribution 4.0 International License, which permits use, sharing, adaptation, distribution and reproduction in any medium or format, as long as you give appropriate credit to the original author(s) and the source, provide a link to the Creative Commons licence, and indicate if changes were made. The images or other third party material in this article are included in the article's Creative Commons licence, unless indicated otherwise in a credit line to the material. If material is not included in the article's Creative Commons licence and your intended use is not permitted by statutory regulation or exceeds the permitted use, you will need to obtain permission directly from the copyright holder. To view a copy of this licence, visit http://creativecommons.org/licenses/by/4.0/ 
fractional Orlicz-Sobolev space $W_{0}^{s} L_{G}(\Omega)$,

$$
(-\Delta)_{g}^{s} u(x)=2 \mathrm{P} . \mathrm{V} \cdot \int_{\Omega} g\left(\frac{|u(x)-u(y)|}{|x-y|^{s}}\right) \frac{u(x)-u(y)}{|u(x)-u(y)|} \frac{d y}{|x-y|^{N+s}}
$$

where P.V. denotes the Cauchy-principle value, $u^{+}=\max \{u, 0\}, u^{-}=-\min \{u, 0\}, \tau \in R$, $a^{(s, g, \alpha)}$ and $b^{(s, g, \alpha)}$ are real numbers depending on $s, g$ and $\alpha$ such that $a^{(s, g, \alpha)}<b^{(s, g, \alpha)}$, and $\phi_{1}^{(s, g, \alpha)}$ is the first eigenfunction, depending on $s, g$ and $\alpha$, of the fractional N-Laplacian eigenvalue problem

$$
\begin{aligned}
& (-\Delta)_{g}^{s} u=\lambda g(|u|) \frac{u}{|u|}, \quad \int_{\Omega} G(|u|) d x=\alpha, \quad \text { in } \Omega, \\
& u=0 \quad \text { on } \partial \Omega,
\end{aligned}
$$

and $W_{0}^{s} L_{G}(\Omega)$ and $\mathrm{N}$-function will be introduced later.

The fractional Orlicz-Sobolev space and the fractional N-Laplacian operators have been studied thoroughly both from the point of view of probability and analysis as they proved to be accurate models to describe different phenomena in Physics, Finance, Image processing and Ecology; see $[2,12,19]$ and references therein; and have been researched by some mathematicians for pure mathematical research and concrete real-world applications in recent years.

Let $g$ be an odd, strictly increasing continuous function from $[0, \infty)$ onto $[0, \infty), g(0)=0$ and $\lim _{t \rightarrow \infty} g(t)=\infty$. The integral representation $\int_{0}^{|t|} g(\tau) d \tau, \forall t \in R$ is an even function with respect to the variable $t \in R$, which is denoted as $G(t)$. Then, the function $G(t)=\int_{0}^{|t|} g(\tau) d \tau, \forall t \in R$ is a Young function and a $\mathrm{N}$-function. A continuous, convex function, $G: R^{+} \rightarrow R^{+}$is a Young function if it is nonnegative, strictly increasing and admits the integral formulation $G(t)=\int_{0}^{t} g(\tau) d \tau, \forall t>0$. (cf. [21]). A continuous, convex function, $G: R \rightarrow R^{+}$is a $\mathrm{N}$-function if it is even and if it satisfies both $\lim _{t \rightarrow 0} \frac{G(t)}{t}=0$ and $\lim _{t \rightarrow \infty} \frac{G(t)}{t}=+\infty$. Equivalently, $G$ is a $\mathrm{N}$-function if and only if there exists a nondecreasing, right continuous function $g:[0, \infty) \rightarrow R^{+}$such that $g(0)=0, g(t)$ is positive for all $t \in(0, \infty), \lim _{t \rightarrow+\infty} g(t)=+\infty$ and $G(t)=\int_{0}^{|t|} g(\tau) d \tau, \forall t \in R$ (cf. [17]). The difference between a Young function and a $\mathrm{N}$-function is that a Young function admits the integral formulation $\int_{0}^{t} g(\tau) d \tau, \forall t>0$, and a N-function is an even function admitting $\int_{0}^{|t|} g(\tau) d \tau$, $\forall t \in R$.

Let $\Omega$ be a bounded domain of $\mathrm{R}^{N}$ with a smooth boundary $\partial \Omega, N \geq 1, s \in(0,1)$ and $p: \Omega \times \Omega \rightarrow(1, \infty)$ be a continuous function with $1<p(x, y)<\infty$. The fractional Sobolev spaces with variable exponent are defined by

$$
W^{s, p(x, y)}(\Omega)=\left\{u \in L^{p(x, x)}(\Omega) \mid \int_{\Omega} \int_{\Omega} \frac{|u(x)-u(y)|^{p(x, y)}}{\lambda^{p(x, y)}|x-y|^{N+s p(x, y)}} d x d y<\infty \text {, for some } \lambda>0\right\} .
$$

Results on the fractional Sobolev spaces with variable exponent and the corresponding nonlocal equations were obtained in [14]. In this paper, we are trying to relax the growth condition on $W^{s, p(x, y)}(\Omega)$ and the corresponding operators, and deal with more generalized spaces and their corresponding operators on the growth condition than the fractional Sobolev spaces and their corresponding operators. When we are trying to relax the growth conditions on the operators, we can not formulate with the fractional Lebesgue spaces and 
the fractional Sobolev spaces $W^{s, p(x, y)}(\Omega)$. We adopt the Orlicz spaces $L_{G}(\Omega)$ associated to a $\mathrm{N}$-function $G$ defined as

$$
\begin{aligned}
L_{G}(\Omega)= & \{u \mid u: \Omega \rightarrow R \text { is a measurable function with } \\
& \left.\|u\|_{L_{G}}=\sup \left\{\int_{\Omega} u v d x \mid \int_{\Omega} G^{*}(|v|) d x \leq 1\right\}<\infty\right\},
\end{aligned}
$$

where $G^{*}$ is the complementary function of $G$, the fractional Orlicz-Sobolev spaces $W^{s} L_{G}(\Omega)$ associated to a $\mathrm{N}$-function $G$ and a fractional parameter $0<s<1$ defined as

$$
\left.W^{s} L_{G} \Omega\right)=\left\{u \in L_{G}(\Omega): \int_{\Omega} \int_{\Omega} G\left(\frac{|u(x)-u(y)|}{|x-y|^{s}}\right) \frac{d x d y}{|x-y|^{N}}<\infty\right\}
$$

and their corresponding nonhomogeneous fractional N-Laplacian operators $(-\Delta)_{g}^{s} u(x)$. From [7], under the conditions that the Young function $G$ and $G^{*}$ satisfy the $\Delta_{2}$ conditions, which is introduced below in this section, for $s \in(0,1), W^{s} L_{G}(\Omega)$ is a reflexine and separable Banach space. Moreover, $C_{0}^{\infty}(\Omega)$ is dense in $W^{s} L_{G}(\Omega)$. We also define $W_{0}^{s} L_{G}(\Omega)$ as the closure of $C_{0}^{\infty}(\Omega)$ in $W^{s} L_{G}(\Omega)$. We refer the readers to $[13,18]$ and the references therein for the theory of Orlicz and Orlicz-Sobolev spaces. We also refer the readers to $[1,4,6,7,20]$ for some results about the fractional Orlicz-Sobolev spaces and the fractional N-Laplacian operator. In [7], the authors provide the connection between the fractional-order theories and the Orlicz-Sobolev ones, and define the fractional-order Orlicz-Sobolev space associated to a Young function and a fractional parameter.

It was proved in $[5,20]$ that under the $\Delta_{2}$ condition on $G$ defined as

$$
G(2 t) \leq C G(t) \quad t \geq T
$$

where $C>0$ and $T \geq 0$, the fractional N-Laplacian eigenvalue problem (1.2) in $W_{0}^{s} L_{G}(\Omega) \cap$ $C(\Omega)$ for each energy level $\alpha>0$ has a discrete nondecreasing sequence of nonnegative eigenvalues $\lambda_{j}^{(s, g, \alpha)}, j=1,2, \ldots$, obtained by the Ljusternik-Schnirelman principle tending to $\infty$ as $j \rightarrow \infty$, and the sequence of the corresponding orthonormalized eigenfunctions $\phi_{j}^{(s, g, \alpha)}, j=1,2, \ldots$. The first eigenvalue $\lambda_{1}^{(s, g, \alpha)}$ is positive and the eigenfunction $\phi_{1}^{(s, g, \alpha)}$ associated with $\lambda_{1}^{(s, g, \alpha)}$ does not change sign, and is positive in $C(\Omega)$ and depends on $s, g$ and $\alpha$. The set of eigenvalues is closed and the first eigenvalue $\lambda_{1}^{(s, g, \alpha)}$ is isolated, which will be proved in Lemma 2.9.

To state our main results we need some properties and give some assumptions: Let $G^{*}$ be the function defined by

$$
G^{*}(t)=\int_{0}^{t} g^{-1}(\tau) d \tau \quad \text { for all } \tau \geq 0
$$

where $g^{-1}$ is the inverse function of $g$. The function $G^{*}$ is called the complementary function of $G$ and satisfies

$$
G^{*}(t)=\sup \{y t-G(y) \mid y \geq 0\} \quad \text { for all } t \geq 0 \text {. }
$$


Then, $G^{*}$ satisfies that

$$
\lim _{t \rightarrow 0} \frac{G^{*}(t)}{t}=0, \quad \lim _{t \rightarrow \infty} \frac{G^{*}(t)}{t}=+\infty,
$$

i.e., $G^{*}$ is a $N$-function. By Young's inequality,

$$
x y \leq G(x)+G^{*}(y), \quad \text { for all } x, y \geq 0 .
$$

For the Young function $G=\int_{0}^{t} g(\tau) d \tau$ let us set

$$
g_{0}=\inf _{t>0} \frac{\operatorname{tg}(t)}{G(t)}, \quad g^{0}=\sup _{t>0} \frac{\operatorname{tg}(t)}{G(t)} .
$$

We assume that

$$
1<g_{0} \leq \frac{\operatorname{tg}(t)}{G(t)} \leq g^{0}<\infty \quad \forall t \geq 0 .
$$

By Proposition 2.3 of [16], it implies that $G$ and $G^{*}$ satisfy the $\Delta_{2}$-condition. We also assume that

$$
\begin{aligned}
& G: t \in[0, \infty) \mapsto G(\sqrt{t}) \quad \text { is convex, } \\
& \int_{0}^{1} \frac{G^{-1} t}{t^{\frac{N+s}{N}}} d t<\infty \quad \text { and } \quad \int_{1}^{\infty} \frac{G^{-1} t}{t^{\frac{N+s}{N}}} d t=\infty, \quad \text { where } 0<s<1 .
\end{aligned}
$$

We also assume that

$$
1<g_{0} \leq g^{0}<g_{0}^{*}=\frac{N g_{0}}{N-s g_{0}} .
$$

An example of a function satisfying (1.6)-(1.8) is $G(t)=t^{p}$ with $2 \leq p \leq \frac{N p}{N-s p}$.

Equation (1.1) is characterized as a jumping problem. A jumping problem was first suggested in the suspension-bridge equation as a model of the nonlinear oscillations in the differential equation

$$
\begin{aligned}
& u_{t t}+K_{1} u_{x x x x}+K_{2} u^{+}=W(x)+\epsilon f(x, t), \\
& u(0, t)=u(L, t)=0, \quad u_{x x}(0, t)=u_{x x}(L, t)=0 .
\end{aligned}
$$

This equation represents a bending beam supported by cables under a load $f$. The constant $K_{2}$ represents the restoring force if the cables stretch. The nonlinearity $u^{+}$models the fact that cables resist expansion but do not resist compression. Choi and Jung (cf. [9-11]) and McKenna and Walter (cf. [15]) investigated the existence and a multiplicity of solutions for the single nonlinear suspension-bridge equation with Dirichlet boundary conditions. In [8], the authors investigated the multiplicity of solutions of a semilinear equation

$$
\begin{aligned}
& A u+b u^{+}-a u^{-}=f(x) \text { in } \Omega, \\
& u=0 \text { on } \Omega,
\end{aligned}
$$


where $\Omega$ is a bounded domain in $\mathrm{R}^{N}, N \geq 1$, with smooth boundary $\partial \Omega$ and $A$ is a secondorder linear partial differential operator when the forcing term is a multiple $s \phi_{1}, s \in R$, of the positive eigenfunction and the nonlinearity crosses eigenvalues.

Since $g$ is an odd, strictly increasing continuous function from $[0, \infty)$ onto $[0, \infty), g(0)=$ 0 and $\lim _{t \rightarrow \infty} g(t)=\infty$, there exists an inverse function $g^{-1}$ of $g$ that is also an odd, continuous function from $[0, \infty)$ onto $[0, \infty), g^{-1}(0)=0$ and $\lim _{t \rightarrow \infty} g^{-1}(t)=\infty$. Then, the function $\tilde{g}: t \mapsto g(|t|) \frac{t}{|t|}$ is an odd, strictly increasing continuous function from $(-\infty, \infty)$ onto $(-\infty, \infty), \tilde{g}(0)=\left.g(|t|) \frac{t}{|t|}\right|_{t=0}=0$ and $\lim _{t \rightarrow \pm \infty} \tilde{g}(t)= \pm \infty$. We note that $\tilde{g}(u)^{+}=g(|u|) \frac{u^{+}}{|u|}$ and $\tilde{g}(u)^{-}=g(|u|) \mid \frac{u^{-}}{|u|}$. Moreover, $\tilde{g}$ has an inverse function $\tilde{g}^{-1}$ that is also an odd, continuous function from $(-\infty, \infty)$ onto $(-\infty, \infty), \tilde{g}^{-1}(0)=0$ and $\lim _{t \rightarrow \infty} \tilde{g}^{-1}(t)=\infty$.

Our main theorems are as follows:

Theorem 1.1 Let $0<s<1, s g_{0}<N, \alpha>0$ and $g$ be an odd, strictly increasing continuous function. We assume that (1.6)-(1.9) hold.

(i) We also assume that $a^{(s, g, \alpha)}<b^{(s, g, \alpha)},-\infty<a^{(s, g, \alpha)}, b^{(s, g, \alpha)}<\lambda_{1}^{(s, g, \alpha)}$.

Then, (1.1) has exactly one nontrivial solution in $W_{0}^{s} L_{G}(\Omega) \cap C(\Omega)$ for all $\tau$ in a bounded interval: In particular, we have that

$$
\begin{aligned}
& \text { if } a^{(s, g, \alpha)}<b^{(s, g, \alpha)},-\infty<a^{(s, g, \alpha)}, b^{(s, g, \alpha)}<\lambda_{1}^{(s, g, \alpha)} \text { and } \tau>0, \text { then } \\
& u=g^{-1}\left(\frac{\tau}{\lambda_{1}^{(s, g, \alpha)}-b^{(s, g, \alpha)}} g\left(\left|\phi_{1}^{(s, g, \alpha)}\right|\right) \frac{\phi_{1}^{(s, g, \alpha)}}{\left|\phi_{1}^{(s, g, \alpha)}\right|}\right)>0 \text { is a solution, } \\
& \text { if } a^{(s, g, \alpha)}<b^{(s, g, \alpha)},-\infty<a^{(s, g, \alpha)}, b^{(s, g, \alpha)}<\lambda_{1}^{(s, g, \alpha)} \text { and } \tau<0, \text { then } \\
& u=-g^{-1}\left(\frac{\tau}{a^{(s, g, \alpha)}-\lambda_{1}^{(s, g, \alpha)}} g\left(\left|\phi_{1}^{(s, g, \alpha)}\right|\right) \frac{\phi_{1}^{(s, g, \alpha)}}{\left|\phi_{1}^{(s, g, \alpha)}\right|}\right)<0 \text { is a solution. }
\end{aligned}
$$

(ii) We also assume that $a^{(s, g, \alpha)}<b^{(s, g, \alpha)}, \lambda_{j}^{(s, g, \alpha)}<a^{(s, g, \alpha)}, b^{(s, g, \alpha)}<\lambda_{j+1}^{(s, g, \alpha)}$ and $\tau \in R$ is bounded, $j=1,2, \ldots$.

Then, (1.1) has exactly one nontrivial solution in $W_{0}^{s} L_{G}(\Omega) \cap C(\Omega)$ for all $\tau$ in the bounded interval.

Theorem 1.2 Let $0<s<1, s g_{0}<N, \alpha>0$ and $g$ be an odd, strictly increasing continuous function. We assume that (1.6)-(1.9) hold. We also assume that $a^{(s, g, \alpha)}<b^{(s, g, \alpha)}$, $-\infty<a^{(s, g, \alpha)}<\lambda_{1}^{(s, g, \alpha)}<b^{(s, g, \alpha)}<\lambda_{2}^{(s, g, \alpha)}, \tau \in R$.

(i) If $\tau>0$, then (1.1) has no solution,

(ii) if $\tau=0$, then (1.1) has exactly one solution $u=0$ in $W_{0}^{s} L_{G}(\Omega) \cap C(\Omega)$.

(iii) There exists $\tau_{1}^{(s, g, \alpha)}<0$ such that for any $\tau$ with $\tau_{1}^{(s, g, \alpha)} \leq \tau<0$, (1.1) has exactly two solutions in $W_{0}^{s} L_{G}(\Omega) \cap C(\Omega)$.

Theorem 1.3 Let $0<s<1$, s $g_{0}<N, \alpha>0$ and $g$ be an odd, strictly increasing continuous function. We assume that (1.6)-(1.9) hold. We also assume that $a^{(s, g, \alpha)}<b^{(s, g, \alpha)}$, $-\infty<a^{(s, g, \alpha)}<\lambda_{1}^{(s, g, \alpha)}, \lambda_{2}^{(s, g, \alpha)}<b^{(s, g, \alpha)}<\lambda_{3}^{(s, g, \alpha)}, \tau \in R$.

(i) If $\tau>0$, then (1.1) has no solution,

(ii) if $\tau=0$, then (1.1) has exactly one solution $u=0$ in $W_{0}^{s} L_{G}(\Omega) \cap C(\Omega)$.

(iii) There exists $\tau_{1}^{(s, g, \alpha)}<0$ such that for any $\tau$ with $\tau_{1}^{(s, g, \alpha)} \leq \tau<0$, (1.1) has at least three solutions in $W_{0}^{s} L_{G}(\Omega) \cap C(\Omega)$.

For the proofs of Theorem 1.1, Theorem 1.2 and Theorem 1.3 we use the contraction mapping principle and Leray-Schauder degree theory on $W_{0}^{s} L_{G}(\Omega) \cap C(\Omega)$. The rest of the paper is organized as follows: In Sect. 2, we introduce some preliminaries and prove Theo- 
rem 1.1 by the direct computations with the eigenvalues and the corresponding eigenfunctions of the fractional N-Laplacian eigenvalue problem, and by the contraction mapping principle on $W_{0}^{s} L_{G}(\Omega) \cap C(\Omega)$. In Sect. 3, we prove Theorem 1.2 by the contraction mapping principle on $W_{0}^{s} L_{G}(\Omega) \cap C(\Omega)$. In Sect. 4, we prove (i), (ii) and (iii) of Theorem 1.3. We prove (iii) of Theorem 1.3 by the Leray-Schauder degree theory on $W_{0}^{s} L_{G}(\Omega) \cap C(\Omega)$.

\section{Preliminaries and proof of Theorem 1.1}

Let $G$ be a $N$-function. From the convexity of $G$, it follows that

$$
G(p t) \leq p G(t) \quad \text { if } p \in[0,1], t \geq 0
$$

and

$$
G(q t) \geq q G(t) \quad \text { if } q \in(1, \infty), t \geq 0
$$

The Orlicz space $L_{G}(\Omega)$ endowed with the norm $\|u\|_{L_{G}}$ is a Banach space. We note that the norm $\|u\|_{L_{G}}$ is equivalent to the Luxemburg norm

$$
\|u\|_{G}=\inf \left\{\lambda>0 \mid \int_{\Omega} G\left(\left|\frac{u(x)}{\lambda}\right|\right) \leq 1\right\} .
$$

In the Orlicz space $L_{G}(\Omega)$, the Hölder inequality is valid (see [18]): for all $u \in L_{G}(\Omega), v \in$ $L_{G^{*}}(\Omega)$, we have

$$
\int_{\Omega}|u v| d x \leq 2\|u\|_{L_{G}}\|v\|_{L_{G^{*}}}
$$

For any given fractional parameter $0<s<1$ and N-function $G$, the fractional OrliczSobolev spaces $W^{s} L_{G}(\Omega)$ is endowed with the norm

$$
\|u\|_{s, G}=\|u\|_{G}+[u]_{s, G}
$$

where $[u]_{s, G}$ is the Gagliardo seminorm defined by

$$
[u]_{s, G}=\inf \left\{\lambda>0 \mid \int_{\Omega} \int_{\Omega} G\left(\frac{|u(x)-u(y)|}{\lambda|x-y|^{s}}\right) \frac{d x d y}{|x-y|^{N}} \leq 1\right\} .
$$

From [7], for any given $0<s<1$ and Young function $G$ such that $G$ and $G^{*}$ satisfy $\Delta_{2}$ conditions, $W^{s} L_{G}\left(R^{N}\right)$ is a reflexive and separable Banach space. Furthermore, $C_{0}^{\infty}\left(R^{N}\right)$ is dense in $W^{s} L_{G}\left(R^{N}\right)$ in the norm $\|\cdot\|_{s, G}$. Let $W_{0}^{s} L_{G}(\Omega)$ denote the closure of $C_{0}^{\infty}(\Omega)$ in the norm $\|u\|_{s, G}$. Later, we shall consider the fractional $\mathrm{N}$-Laplacian eigenvalue problem (1.2) in $W_{0}^{s} L_{G}(\Omega) \cap C(\Omega)$ under the $\Delta_{2}$ condition on $G$ and prove Theorem 1.1.

Lemma 2.1 ([20] (Generalized Poincaré inequality on the Orlicz-Sobolev space)) Let $\Omega$ be a bounded open subset of $R^{N}, N \geq 1,0<s<1$ and $G$ be a Young function. Then, there exists a positive constant $C>0$ such that

$$
\|u\|_{G} \leq C[u]_{s, G}, \quad \forall u \in W_{0}^{s} L_{G}(\Omega) .
$$


That is, the embedding

$$
W_{0}^{s} L_{G}(\Omega) \hookrightarrow L_{G}(\Omega)
$$

is continuous and compact. Furthermore, $[u]_{s, G}$ is a norm of $W_{0}^{s} L_{G}(\Omega)$ equivalent to $\|\cdot\|_{s, G}$.

Lemma 2.2 ((Theorem 2.8 of [4]) (Embedding Theorem)) Let $\Omega$ be a bounded domain of $R^{N}, N \geq 1,0<s<1$ and $G$ be a $N$-function. Then, the embedding

$$
W^{s} L_{G}(\Omega) \hookrightarrow L_{G}(\Omega) \quad \text { is continuous and compact }
$$

and there exists a positive constant $D>0$ such that

$$
\|u\|_{G} \leq D[u]_{s, G}, \quad \forall u \in W^{s} L_{G}(\Omega) .
$$

Furthermore, $[u]_{s, G}$ is a norm of $W^{s} L_{G}(\Omega)$ equivalent to $\|u\|_{s, G}$.

Let us define the functional $\Psi_{s, G}: W^{s} L_{G}(\Omega) \rightarrow R$ as

$$
\Psi_{s, G}(u)=\int_{\Omega} \int_{\Omega} G\left(\frac{|u(x)-u(y)|}{|x-y|^{s}}\right) \frac{d x d y}{|x-y|^{N}}, \quad u \in W^{s} \cdot L_{G}(\Omega) .
$$

Lemma $2.3([3])$ Let $u \in W^{s} L_{G}(\Omega)$. Then,

$$
\begin{aligned}
& \|u\|_{s, G}^{g_{0}} \leq \Psi_{s, G}(u)=\int_{\Omega} \int_{\Omega} G\left(\frac{|u(x)-u(y)|}{|x-y|^{s}}\right) \frac{d x d y}{|x-y|^{N}} \leq\|u\|_{s, G}^{g^{0}}, \quad \text { if }\|u\|_{s, G}>1, \\
& \|u\|_{s, G}^{g^{0}} \leq \Psi_{s, G}(u)=\int_{\Omega} \int_{\Omega} G\left(\frac{|u(x)-u(y)|}{|x-y|^{s}}\right) \frac{d x d y}{|x-y|^{N}} \leq\|u\|_{s, G}^{g_{0}}, \quad \text { if }\|u\|_{s, G}<1 .
\end{aligned}
$$

Proof The proof is given by (1.4) and Theorem 3.11 of [3].

Lemma 2.4 ([21]) Let $0<s<1, s g_{0}<N$ and $G$ be a $N$-function. Then, the embedding

$$
W^{s, g_{0}}(\Omega) \hookrightarrow L^{q(x)}(\Omega)
$$

is continuous and compact for all $1 \leq q(x)<g_{0}^{*}=\frac{N g_{0}}{N-s g_{0}}$.

Lemma 2.5 Let $0<s<1, s g_{0}<N$ and $G$ be a $N$-function. Then, the embedding

$$
W^{s} L_{G}(\Omega) \hookrightarrow L^{q(x)}(\Omega)
$$

is continuous and compact for all $1 \leq q(x)<g_{0}^{*}$.

Furthermore, there exists a positive constant $C$ such that

$$
\|u\|_{L^{q(x)}(\Omega)} \leq C[u]_{s, G} \text { for all } 1 \leq q(x)<g_{0}^{*} .
$$


Proof By Lemma 2.4, the embedding $W^{s, g_{0}}(\Omega) \hookrightarrow L^{q(x)}(\Omega)$ is continuous and compact for all $1 \leq q(x)<g_{0}^{*}=\frac{N g_{0}}{N-s g_{0}}$. By (1.6) and (2.3), the embedding $W^{s} L_{G}(\Omega) \hookrightarrow W^{s, g_{0}}(\Omega)$ is continuous. Combining these facts, we obtain that the embedding $W^{s} L_{G}(\Omega) \hookrightarrow L^{q(x)}(\Omega)$ is continuous and compact for all $1 \leq q(x)<g_{0}^{*}=\frac{N g_{0}}{N-s g_{0}}$.

Lemma 2.6 Assume that the sequence $\left\{u_{k}\right\}$ converges weakly to $u$ in $W^{s} L_{G}(\Omega)$ and

$$
\lim _{k \rightarrow+\infty} \sup <\Psi_{s, G}^{\prime}\left(u_{k}\right), \quad u_{k}-u>\leq 0 .
$$

Then, $\left\{u_{k}\right\}$ converges strongly to $u$ in $W^{s} L_{G}(\Omega)$.

Proof Since the sequence $\left\{u_{k}\right\}$ converges weakly to $u$ in $W^{s} L_{G}(\Omega)$ and $\lim _{k \rightarrow+\infty}$ sup $<$ $\Psi^{\prime}\left(u_{k}\right), u_{k}-u>\leq 0$, by (1.6), we have

$$
\begin{aligned}
& \int_{\Omega} \int_{\Omega} g\left(\frac{\left|u_{k}(x)-u_{k}(y)\right|}{|x-y|^{s}}\right) \frac{u_{k}(x)-u_{k}(y)}{\left|u_{k}(x)-u_{k}(y)\right|} \frac{u_{k}(x)-u_{k}(y)}{|x-y|^{s}} \frac{d x d y}{|x-y|^{N}} \\
& \quad \leq \int_{\Omega} \int_{\Omega} g\left(\frac{|u(x)-u(y)|}{|x-y|^{s}}\right) \frac{u(x)-u(y)}{|u(x)-u(y)|} \frac{u(x)-u(y)}{|x-y|^{s}} \frac{d x d y}{|x-y|^{N}} \\
& \quad \leq g^{0} \int_{\Omega} \int_{\Omega} G\left(\frac{|u(x)-u(y)|}{|x-y|^{s}}\right) \frac{d x d y}{|x-y|^{N}} .
\end{aligned}
$$

Thus, the sequence $\left\{\int_{\Omega} \int_{\Omega} g\left(\frac{\left|u_{k}(x)-u_{k}(y)\right|}{|x-y|^{s}}\right) \frac{u_{k}(x)-u_{k}(y)}{\left|u_{k}(x)-u_{k}(y)\right|} \frac{u_{k}(x)-u_{k}(y)}{|x-y|^{s}} \frac{d x d y}{|x-y|^{N}}\right\}$ is bounded and converges to $\int_{\Omega} \int_{\Omega} g\left(\frac{|u(x)-u(y)|}{|x-y|^{s}}\right) \frac{u(x)-u(y)}{|u(x)-u(y)|} \frac{u(x)-u(y)}{|x-y|^{s}} \frac{d x d y}{|x-y|^{N}}$. By (1.6), we have

$$
\begin{aligned}
& \int_{\Omega} \int_{\Omega} g\left(\frac{\left|u_{k}(x)-u_{k}(y)\right|}{|x-y|^{s}}\right) \frac{u_{k}(x)-u_{k}(y)}{\left|u_{k}(x)-u_{k}(y)\right|} \frac{u_{k}(x)-u_{k}(y)}{|x-y|^{s}} \frac{d x d y}{|x-y|^{N}} \\
& \quad \geq g_{0} \int_{\Omega} \int_{\Omega} G\left(\frac{\left|u_{k}(x)-u_{k}(y)\right|}{|x-y|^{s}}\right) \frac{d x d y}{|x-y|^{N}} .
\end{aligned}
$$

Thus, the sequence $\left\{\int_{\Omega} \int_{\Omega} G\left(\frac{\left|u_{k}(x)-u_{k}(y)\right|}{|x-y|^{s}}\right) \frac{d x d y}{|x-y|^{N}}\right\}$ is bounded and converges to $\int_{\Omega} \int_{\Omega} G\left(\frac{|u(x)-u(y)|}{|x-y|^{s}}\right) \frac{d x d y}{|x-y|^{N}}$. Thus, the sequence $\left\{u_{k}\right\}$ is bounded and converges weakly to $u$ in $W^{s} L_{G}(\Omega)$. Since the embedding $W^{s} L_{G}(\Omega) \hookrightarrow L_{G}(\Omega)$ is continuous and compact, $\left\{u_{k}\right\}$ converges strongly to $u$ in $W^{s} L_{G}(\Omega)$.

Lemma 2.7 If $u_{k}, u \in W^{s} L_{G}(\Omega), k=1,2, \ldots$, then the following statements are equivalent to each other

(i) $\lim _{k \rightarrow \infty}\left\|u_{k}-u\right\|_{s, G}=0, k=1,2$,

(ii) $\lim _{k \rightarrow \infty} \int_{\Omega}\left(G\left(u_{k}(x)-u(x)\right) d x=0\right.$ and $\lim _{k \rightarrow \infty}\left[u_{k}-u\right]_{s, G}=0$,

(iii) $\lim _{k \rightarrow \infty} \int_{\Omega} G\left(u_{k}(x)\right) d x=\int_{\Omega} G(u(x)) d x$.

Proof By the definition of $\|\cdot\|_{s, G}$, (i) $\Leftrightarrow$ (ii) holds. We shall show that (i) implies (iii). We assume that (i) holds. Then,

$$
\begin{aligned}
& \int_{\Omega}\left[G\left(u_{k}\right)(x)-G(u)(x)\right] d x \\
& \quad \leq \int_{\Omega} g\left(u+\lambda\left(u_{k}-u\right)\right)\left(u_{k}-u\right) d x
\end{aligned}
$$




$$
\begin{aligned}
& \leq 2\left\|g\left(u+\lambda\left(u_{k}-u\right)\right)\right\|_{G^{*}}\left\|u_{k}-u\right\|_{G} \\
& \leq C\left\|g\left(u+\lambda\left(u_{k}-u\right)\right)\right\|_{G^{*}}\left\|u_{k}-u\right\|_{s, G} \rightarrow 0
\end{aligned}
$$

for $0<\lambda<1$ and some $C>0$. It follows that (iii) holds. Assume that (iii) holds. Since $\lim _{k \rightarrow \infty} \int_{\Omega} G\left(u_{k}(x)\right) d x=\int_{\Omega} G(u(x)) d x,\left\{u_{k}\right\}$ converges weakly to $u$ in $L_{G}(\Omega)$. By assumption (iii), $u_{k} \rightarrow u$ in measure in $W^{s} L_{G}(\Omega)$. It follows that $\left\{u_{k}\right\}$ is bounded in $W^{s} L_{G}(\Omega) \subset$ $L_{G}(\Omega)$. By Lemma 2.1, the embedding $W^{s} L_{G}(\Omega) \hookrightarrow L_{G}(\Omega)$ is continuous and compact. Thus, $u_{k} \rightarrow u$ strongly in $W^{s} L_{G}(\Omega)$. Thus, (i) holds.

By Lemma 2.1, we obtain the following:

Lemma 2.8 Let $0<s<1, G$ be a $N$-function and $f(x, u) \in L_{G}(\Omega)$. Then, the solutions of the problem

$$
\begin{aligned}
& (-\Delta)_{g}^{s} u=f(x, u) \quad \text { in } L_{G}(\Omega), \\
& u=0 \quad \partial \Omega
\end{aligned}
$$

belong to $W_{0}^{s} L_{G}(\Omega)$.

Now, we consider the fractional N-Laplacian eigenvalue problem (1.2) in $W_{0}^{s} L_{G}(\Omega) \cap$ $C(\Omega)$ under the conditions (1.6)-(1.9) on $G$ for each energy level $\alpha>0$ :

$$
\begin{aligned}
& (-\Delta)_{g}^{s} u=\lambda g(|u|) \frac{u}{|u|}, \quad \int_{\Omega} G(|u|) d x=\alpha, \quad \text { in } \Omega . \\
& u=0 \quad \text { on } \partial \Omega .
\end{aligned}
$$

Lemma 2.9 (The fractional N-Laplacian eigenvalue problem) Let $\alpha>0$ be a real number and $G$ be a N-function. We assume that (1.6)-(1.9) hold. Then,

(i) the fractional $N$-Laplacian eigenvalue problem (1.2) in $W_{0}^{s} L_{G}(\Omega) \cap C(\Omega)$ has a discrete nondecreasing sequence of nonnegative eigenvalues $\lambda_{j}^{(s, g, \alpha)}, \lambda_{j}^{(s, g, \alpha)} \rightarrow \infty$ as $j \rightarrow \infty, j=1,2, \ldots$ and

(ii) a sequence of the corresponding eigenfunctions $u_{j}^{(s, g, \alpha)}, j=1,2, \ldots$, depending on $s, g$, a. Moreover,

(iii) we can construct the orthonormalized corresponding eigenfunctions $\phi_{j}^{(s, g, \alpha)}$ from the corresponding eigenfunctions $u_{j}^{(s, g, \alpha)}$ belonging to the eigenvalues $\lambda_{j}^{(s, g, \alpha)}, j=1,2, \ldots$,

(iv) the first eigenvalue $\lambda_{1}^{(s, g, \alpha)}$ is positive,

(v) the set of eigenvalues is closed,

(vi) the first eigenfunction $\phi_{1}^{(s, g, \alpha)}$ is positive and

(vii) the first eigenvalue $\lambda_{1}^{(s, g, \alpha)}$ is isolated.

Proof (i) and (ii) are proved in Theorem 1.2 of [5].

(iii) By (i) and (ii), the eigenvalues $\lambda_{j}^{(s, g, \alpha)}, j=1,2, \ldots$ are discrete. Then, the corresponding eigenfunctions $u_{j}^{(s, g, \alpha)}, j=1,2, \ldots$, are mutually orthogonal, i.e., $\operatorname{span}\left\{u_{j}^{(s, g, \alpha)}\right\} \perp \operatorname{span}\left\{u_{k}^{(s, g, \alpha)}\right\}$ for $j \neq k, j, k=1,2, \ldots$ That is, $u_{j}^{(s, g, \alpha)}, j=1,2, \ldots$ are the orthogonal eigenfunctions. Let us 
set

$$
\phi_{j}^{(s, g, \alpha)}=\frac{u_{j}^{(s, g, \alpha)}}{\left\|u_{j}^{(s, g, \alpha)}\right\|_{s, G}}, \quad j=1,2, \ldots
$$

Then, $\phi_{j}^{(s, g, \alpha)}, j=1,2, \ldots$ are the orthonormalized eigenfunctions belonging to the eigenvalues $\lambda_{j}^{(s, g, \alpha)}, j=1,2, \ldots$.

(iv) (iv) is proved in Corollary 5.3 of [20].

(v) (v) is proved in Theorem 1.5 of [5].

(vi) Since the functionals $\int_{\Omega} \int_{\Omega} G\left(\frac{|u(x)-u(y)|}{\lambda|x-y|^{s}}\right) \frac{d x d y}{|x-y|^{N}}$ and $\int_{\Omega} G(|u|) d x$ are invariant by replacing $\phi_{1}^{(s . g, \alpha)}$ with $\left|\phi_{1}^{(s . g, \alpha)}\right|, \phi_{1}^{(s . g, \alpha)} \geq 0$. Since (1.2) is considered in $W_{0}^{s} L_{G}(\Omega) \cap C(\Omega)$, $\phi_{1}^{(s . g, \alpha)} \in W_{0}^{s} L_{G}(\Omega) \cap C(\Omega)$. By the strong maximum principle for continuous functions, $\phi_{1}^{(s . g, \alpha)}$ is positive in $\Omega$ (cf. Proposition 3.8 of [5]).

(vii) To show the isolatedness of the first eigenvalue, we shall show the process of obtaining the first eigenvalue associated with the first eigenfunction and the other eigenvalues associated with the other eigenfunctions. We say that $\lambda$ is an eigenvalue with eigenfunction $\phi \in W_{0}^{s} L_{G}(\Omega)$ if

$$
<(-\Delta)_{g}^{s} \phi, v>-\lambda \int_{\Omega} g(|\phi|) \frac{\phi}{|\phi|} v d x=0 \quad \forall v \in W_{0}^{s} L_{G}(\Omega) .
$$

For each $\lambda>0$ let us define the energy functional $\Phi_{\lambda}^{(s, g, \alpha)}: W_{0}^{s} L_{G}(\Omega) \cap C(\Omega) \rightarrow R$ associated to $(1.2)$ as

$$
\begin{aligned}
& \Phi_{\lambda}^{(s, g, \alpha)}(u)=\int_{\Omega} \int_{\Omega} G\left(\frac{|u(x)-u(y)|}{|x-y|^{s}}\right) \frac{d x d y}{|x-y|^{N}}-\lambda \int_{\Omega} G(|u|) d x \\
& \int_{\Omega} G(|u|) d x=\alpha, \quad \alpha>0 .
\end{aligned}
$$

Under the $\Delta_{2}$-condition on $G$ the functional $\Phi_{\lambda}^{(s, g, \alpha)}(u)$ is Fréchet differentiable, $C^{1}$ and satisfies the Palaise-Smale condition. The critical points of $\Phi_{\lambda}^{(s, g, \alpha)}(u)$ coincide with the weak solutions of (1.2). We claim that for each $\lambda>0$ the functional $\Phi_{\lambda}^{(s, g, \alpha)}(u)$ is coercive and weakly lower semicontinuous. In fact, by Lemma 3.2 and Lemma 3.3 of [6], $\int_{\Omega} \int_{\Omega} G\left(\frac{|u(x)-u(y)|}{|x-y|^{s}}\right) \frac{d x d y}{|x-y|^{N}}$ is of class $C^{1}$ and weakly lower semicontinuous. Let $u \in W_{0}^{s} L_{G}(\Omega)$ with $\|u\|_{s, G}>1$. By Lemma 2.2, there exists a constant $D>0$ such that $\|u\|_{G} \leq D\|u\|_{s, G}$. It follows from Lemma 2.3 that

$$
\begin{aligned}
\Phi_{\lambda}^{(s, g, \alpha)}(u) & =\int_{\Omega} \int_{\Omega} G\left(\frac{|u(x)-u(y)|}{|x-y|^{s}}\right) \frac{d x d y}{|x-y|^{N}}-\lambda \int_{\Omega} G(|u|) d x \\
& \geq\|u\|_{(s, G)}^{g_{0}}-\lambda\|u\|_{G} \\
& \geq\|u\|_{(s, G)}^{g_{0}}-\lambda D\|u\|_{s, G} .
\end{aligned}
$$

Since $g_{0}>1, \Phi_{\lambda}^{(s, g, \alpha)}(u) \rightarrow \infty$ as $\|u\|_{s, G} \rightarrow \infty$. Thus, $\Phi_{\lambda}^{(s, g, \alpha)}(u)$ is coercive.

Next, we shall show that $\Phi_{\lambda}^{(s, g, \alpha)}(u)$ is weakly lower semicontinuous. Let $u_{n} \in W_{0}^{s} L_{G}(\Omega)$ be a sequence that converges weakly to $u$ in $W_{0}^{s} L_{G}(\Omega)$. By Lemma 3.3 of [6],

$$
\int_{\Omega} \int_{\Omega} G\left(\frac{|u(x)-u(y)|}{|x-y|^{s}}\right) \frac{d x d y}{|x-y|^{N}} \leq \lim _{n \rightarrow+\infty} \inf \int_{\Omega} \int_{\Omega} G\left(\frac{\left|u_{n}(x)-u_{n}(y)\right|}{|x-y|^{s}}\right) \frac{d x d y}{|x-y|^{N}}
$$


On the other hand, since $G$ is a continuous function,

$$
\lim _{n \rightarrow+\infty} \int_{\Omega} G\left(\left|u_{n}\right|\right) d x=\int_{\Omega} G(|u|) d x
$$

Thus, we have

$$
\Phi_{\lambda}^{(s, g, \alpha)}(u) \leq \lim _{n \rightarrow+\infty} \inf \Phi_{\lambda}^{(s, g, \alpha)}\left(u_{n}\right) .
$$

Thus, $\Phi_{\lambda}^{(s, g, \alpha)}$ is weakly lower semicontinuous. It follows that there exists only one global minimizer $\phi_{1}^{(s, g, \alpha)} \in W_{0}^{s} L_{G}(\Omega)$, which is a critical point of $\Phi_{\lambda}^{(s, g, \alpha)}(u)$ with a global minimum value $\inf _{W_{0}^{s} L_{G}(\Omega) \cap C(\Omega)} \Phi_{\lambda}^{(s, g, \alpha)}(t v) \leq\|t v\|_{s, G}^{g^{0}}-\lambda_{1}^{(s, g, \alpha)} \alpha<0$ for sufficiently small $t>0$ and $g^{0}>1$, nonnegative, and positive in $C(\Omega)$ by (vi). Let us set $\lambda=\lambda_{1}^{(s, g, \alpha)}$ for the eigenvalue corresponding to the weak solution $\phi_{1}^{(s, g, \alpha)}$ of the problem

$$
(-\Delta)_{g}^{s} \phi_{1}^{(s, g, \alpha)}=\lambda g\left(\left|\phi_{1}^{(s, g, \alpha)}\right|\right) \frac{\phi_{1}^{(s, g, \alpha)}}{\left|\phi_{1}^{(s, g, \alpha)}\right|} .
$$

Then, by (iv), $\lambda_{1}^{(s, g, \alpha)}>0$. By Theorem 1.2 of [5] and (iii), (1.2) has the other weak solutions $\phi_{j}^{(s, g, \alpha)}, j \geq 2$, different from $\phi_{1}^{(s, g, \alpha)}$. In fact, for each $\alpha>0$ the other weak solutions $\phi_{j}^{(s, g, \alpha)}$, $j \geq 2$ of (1.2) are obtained from the minimax theory. That is, for any $\alpha>0$ there exists a discrete sequence of nonnegative eigenvalues $\lambda_{j}^{(s, g, \alpha)}$. The corresponding eigenfunctions $\left\{\phi_{j}^{(s, g, \alpha)}\right\} \subset W_{0}^{s} L_{G}(\Omega)$ satisfy the constraint

$$
\int_{\Omega} G\left(\left|\phi_{j}^{(s, g, \alpha)}\right|\right) d x=\alpha, \quad \int_{\Omega} \int_{\Omega} G\left(\frac{\left|\phi_{j}^{(s, g, \alpha)}(x)-\phi_{j}^{(s, g, \alpha)}(y)\right|}{|x-y|^{s}}\right) \frac{d x d y}{|x-y|^{N}}=c_{j}^{(s, g, \alpha)},
$$

where the critical values are obtained as

$$
c_{j}^{(s, g, \alpha)}=\inf _{h \in \Gamma\left(S^{j-1}, M_{\alpha}\right)} \sup _{w \in S^{j-1}} \int_{\Omega} \int_{\Omega} G\left(\frac{|h(w(x))-h(w(y))|}{|x-y|^{s}}\right) \frac{d x d y}{|x-y|^{N}}
$$

and $\Gamma\left(S^{j}, M_{\alpha}\right)=\left\{h \in C\left(S^{j}, M_{\alpha}\right) \mid h\right.$ is odd $\}$, being $S^{j}$ the unit sphere in $R^{j+1}$. The first eigenfunction $\phi_{1}^{(s, g, \alpha)}$ is a global minimizer and the other eigenfunctions $\phi_{j}^{(s, g, \alpha)}, j=2,3, \ldots$ are critical points obtained from the minimax critical values $\Phi_{\lambda}^{(s, g, \alpha)}\left(\phi_{j}^{(s, g, \alpha)}\right)$. As the first eigenfunction $\phi_{1}^{(s, g, \alpha)}$ can not be the critical points obtained from the minimax critical value, $\phi_{1}^{(s, g, \alpha)} \neq \phi_{j}^{(s, g, \alpha)}, j=2,3, \ldots$ Thus, the first eigenvalue $\lambda_{1}^{(s, g, \alpha)}$ corresponding to the eigenfunction $\phi_{1}^{(s, g, \alpha)}$ is isolated from the other eigenvalues $\lambda_{j}^{(s, g, \alpha)}, j=2,3, \ldots$ corresponding to the eigenfunctions $\phi_{j}^{(s, g, \alpha)}, j=2,3, \ldots$

Let $I: f \in C(\Omega \times \Omega) \rightarrow R$ be an integral operator defined as

$$
I(f(x, y))=2 \text { P.V. } \int_{\Omega} f(x, y) \frac{d y}{|x-y|^{N+s}} .
$$

Then, $(-\Delta)_{g}^{s} u$ can be expressed as the composition of the mappings $I, \tilde{g}$ and $D_{s}$ as follows:

$$
(-\Delta)_{g}^{s} u=\left(I \circ \tilde{g} \circ D_{s} \circ \tilde{g}^{-1}\right)(\tilde{g}(u))
$$


or

$$
(\Delta)_{g}^{s} u=\left(I \circ \tilde{g} \circ D_{s} \circ g^{-1}\right)(g(u)),
$$

where $D_{s} u(x, y)=\frac{u(x)-u(y)}{|x-y|^{s}}$ and $\tilde{g}(u)=g(|u|) \frac{u}{|u|}$. We note that the operator norm of $(I \circ \tilde{g} \circ$ $\left.D_{s} \circ \tilde{g}^{-1}\right)^{-1}$ is

$$
\left\|\left(I \circ \tilde{g} \circ D_{s} \circ \tilde{g}^{-1}\right)^{-1}\right\|=\frac{1}{\lambda_{1}^{(s, g, \alpha)}} .
$$

For given $f(x, u) \in L_{G}(\Omega)$, the equation

$$
(-\Delta)_{g}^{s} u=f(x, u) \quad \text { in } L_{G}(\Omega)
$$

is equivalent to the equation

$$
u=\left((-\Delta)_{g}^{s}\right)^{-1} f(x, u)
$$

We observe that

$$
\left\|\left((-\Delta)_{g}^{s}\right)^{-1} f(x, u)\right\|_{s, G} \leq\left\|\tilde{g}^{-1}\left(\frac{1}{\lambda_{1}^{(s, g, \alpha)}} f(x, u)\right)\right\|_{s, G} .
$$

Proof of Theorem 1.1 (i) Let $0<s<1, G$ be a N-function and $\int_{\Omega} G(|u(x)|) d x=\alpha, u \in$ $W_{0}^{s} L_{G}(\Omega) \cap C(\Omega)$. We assume that $-\infty<a^{(s, g, \alpha)}, b^{(s, g, \alpha)}<\lambda_{1}^{(s, g, \alpha)}$. Let us choose $v^{(s, g, \alpha)}>0$ and $\epsilon^{(s, g, \alpha)}>0$ so that $-v^{(s, g, \alpha)}+\epsilon^{(s, g, \alpha)}<a^{(s, g, \alpha)}, b^{(s, g, \alpha)}<\lambda_{1}^{(s, g, \alpha)}-\epsilon^{(s, g, \alpha)}$ and choose $\delta^{(s, g, \alpha)}=$ $\frac{a^{(s, g, \alpha)}+b^{(s, g, \alpha)}}{2}$. Then, the problem (1.1) can be rewritten as

$$
\begin{aligned}
(-\Delta)_{g}^{s} u-\delta^{(s, g, \alpha)} g(|u|) \frac{u}{|u|}= & \left(b^{(s, g, \alpha)}-\delta^{(s, g, \alpha)}\right) g(|u|) \frac{u^{+}}{|u|}-\left(a^{(s, g, \alpha)}-\delta^{(s, g, \alpha)}\right) g(|u|) \frac{u^{-}}{|u|} \\
& +\tau g\left(\left|\phi_{1}^{(s, g, \alpha)}\right|\right) \frac{\phi_{1}^{(s, g, \alpha)}}{\left|\phi_{1}^{(s, g, \alpha)}\right|} .
\end{aligned}
$$

Since $(-\Delta)_{g}^{s} u=\left(I \circ \tilde{g} \circ D_{s} \circ \tilde{g}^{-1}\right)(\tilde{g}(u)),(1.1)$ can be rewritten as

$$
\begin{aligned}
\left(I \circ \tilde{g} \circ D_{s} \circ \tilde{g}^{-1}-\delta^{(s, g, \alpha)}\right) \tilde{g}(u)= & \left(b^{(s, g, \alpha)}-\delta^{(s, g, \alpha)}\right) \tilde{g}(u)^{+}-\left(a^{(s, g, \alpha)}-\delta^{(s, g, \alpha)}\right) \tilde{g}(u)^{-} \\
& +\tau g\left(\left|\phi_{1}^{(s, g, \alpha)}\right|\right) \frac{\phi_{1}^{(s, g, \alpha)}}{\left|\phi_{1}^{(s, g, \alpha)}\right|} .
\end{aligned}
$$

or equivalently

$$
\begin{aligned}
\tilde{g}(u)= & \left(I \circ \tilde{g} \circ D_{s} \circ \tilde{g}^{-1}-\delta^{(s, g, \alpha)}\right)^{-1}\left(\left(b^{(s, g, \alpha)}-\delta^{(s, g, \alpha)}\right) \tilde{g}(u)^{+}-\left(a^{(s, g, \alpha)}-\delta^{(s, g, \alpha)}\right) \tilde{g}(u)^{-}\right. \\
& \left.+\tau g\left(\left|\phi_{1}^{(s, g, \alpha)}\right|\right) \frac{\phi_{1}^{(s, g, \alpha)}}{\left|\phi_{1}^{(s, g, \alpha)}\right|}\right) .
\end{aligned}
$$


The operator norm of the operator $\left(I \circ \tilde{g} \circ D_{s} \circ \tilde{g}\right)^{-1}-\delta^{(s, g, \alpha)^{-1}}$ is

$$
\left\|\left(I \circ \tilde{g} \circ D_{s} \circ \tilde{g}^{-1}-\delta^{(s, g, \alpha)}\right)^{-1}\right\|=\frac{1}{\lambda_{1}^{(s, g, \alpha)}-\delta^{(s, g, \alpha)}} .
$$

Thus, we have that

$$
\begin{aligned}
& \left(I \circ \tilde{g} \circ D_{s} \circ \tilde{g}^{-1}-\delta^{(s, g, \alpha)}\right)^{-1}\left(\left(b^{(s, g, \alpha)}-\delta^{(s, g, \alpha)}\right) \tilde{g}(u)^{+}-\left(a^{(s, g, \alpha)}-\delta^{(s, g, \alpha)}\right) \tilde{g}(u)^{-}\right. \\
& \left.\quad+\tau g\left(\left|\phi_{1}^{(s, g, \alpha)}\right|\right) \frac{\phi_{1}^{(s, g, \alpha)}}{\left|\phi_{1}^{(s, g, \alpha)}\right|}\right) \\
& \leq \frac{1}{\lambda_{1}^{(s, g, \alpha)}-\delta^{(s, g, \alpha)}} \|\left(b^{(s, g, \alpha)}-\delta^{(s, g, \alpha)}\right) \tilde{g}(u)^{+}-\left(a^{(s, g, \alpha)}-\delta^{(s, g, \alpha)}\right) \tilde{g}(u)^{-} \\
& \quad+\tau g\left(\left|\phi_{1}^{(s, g, \alpha)}\right|\right) \frac{\phi_{1}^{(s, g, \alpha)}}{\left|\phi_{1}^{(s, g, \alpha)}\right|} \|_{G}
\end{aligned}
$$

Let us set the right-hand side of (2.6) as

$$
\begin{aligned}
M^{(s, g, \alpha)}\left(g(|u|) \frac{u}{|u|}\right)= & M^{(s, g, \alpha)}(\tilde{g}(u)) \\
= & \left(I \circ \tilde{g} \circ D_{s} \circ \tilde{g}^{-1}-\delta^{(s, g, \alpha)}\right)^{-1}\left(\left(b^{(s, g, \alpha)}-\delta^{(s, g, \alpha)}\right) \tilde{g}(u)^{+}\right. \\
& \left.-\left(a^{(s, g, \alpha)}-\delta^{(s, g, \alpha)}\right) \tilde{g}(u)^{-}+\tau g\left(\left|\phi_{1}^{(s, g, \alpha)}\right|\right) \frac{\phi_{1}^{(s, g, \alpha)}}{\left|\phi_{1}^{(s, g, \alpha)}\right|}\right) .
\end{aligned}
$$

Then, $M^{(s, g, \alpha)}\left(g(|u|) \frac{u}{|u|}\right)$ satisfies

$$
\begin{aligned}
\| & M^{(s, g, \alpha)}(\tilde{g}(u))-M^{(s, g, \alpha)}(\tilde{g}(v)) \|_{s, G} \\
= & \|\left(I \circ \tilde{g} \circ D_{s} \circ \tilde{g}^{-1}-\delta^{(s, g, \alpha)}\right)^{-1}\left(\left(b^{(s, g, \alpha)}-\delta^{(s, g, \alpha)}\right) \tilde{g}(u)^{+}-\left(a^{(s, g, \alpha)}-\delta^{(s, g, \alpha)}\right) \tilde{g}(u)^{-}\right) \\
& \quad-\left(I \circ \tilde{g} \circ D_{s} \circ \tilde{g}^{-1}-\delta^{(s, g, \alpha)}\right)^{-1}\left(\left(b^{(s, g, \alpha)}-\delta^{(s, g, \alpha)}\right) \tilde{g}(v)^{+}-\left(a^{(s, g, \alpha)}-\delta^{(s, g, \alpha)}\right) \tilde{g}(v)^{-}\right) \|_{s, G} \\
\leq & \frac{1}{\lambda_{1}^{(s, g, \alpha)}-\delta^{(s, g, \alpha)}} \|\left(\left(b^{(s, g, \alpha)}-\delta^{(s, g, \alpha)}\right) \tilde{g}(u)^{+}-\left(a^{(s, g, \alpha)}-\delta^{(s, g, \alpha)}\right) \tilde{g}(u)^{-}\right) \\
& -\left(\left(b^{(s, g, \alpha)}-\delta^{(s, g, \alpha)}\right) \tilde{g}(v)^{+}-\left(a^{(s, g, \alpha)}-\delta^{(s, g, \alpha)}\right) \tilde{g}(v)^{-}\right) \|_{G} \\
\leq & \frac{1}{\lambda_{1}^{(s, g, \alpha)}-\delta^{(s, g, \alpha)}} \frac{b^{s, g, \alpha}-a^{(s, g, \alpha)}}{2}\|\tilde{g}(u)-\tilde{g}(v)\|_{G} .
\end{aligned}
$$

Since $\frac{1}{\lambda_{1}^{(s, g, \alpha)}-\delta^{(s, g, \alpha)}} \frac{b^{s, g, \alpha}-a^{(s, g, \alpha)}}{2}<1, M^{(s, g, \alpha)}$ is a contraction mapping with respect to the variable $\tilde{g}(u)$. Thus, (2.6) has a unique solution $\tilde{g}(u)$ for each $s, g, \alpha$. Since $\tilde{g}(u)$ is a strictly increasing continuous function, then there exists a unique $u$ for a unique given value $\tilde{g}(u)$. Thus, (1.1) has a unique solution $u \neq 0$.

(ii) We assume that $\lambda_{j}^{(s, g, \alpha)}<a^{(s, g, \alpha)}, b^{(s, g, \alpha)}<\lambda_{j+1}^{(s, g, \alpha)}, j=1,2, \ldots$ Let us choose $\epsilon^{(s, g, \alpha)}>0$ so that $\lambda_{j}^{(s, g, \alpha)}+\epsilon^{(s, g, \alpha)}<a^{(s, g, \alpha)}, b^{(s, g, \alpha)}<\lambda_{j+1}^{(s, g, \alpha)}-\epsilon^{(s, g, \alpha)}$. Let us set $\gamma^{(s, g, \alpha)}=\frac{a^{(s, g, \alpha)}+b^{(s, g, \alpha)}}{2}$. Then, 
the problem (1.1) can be rewritten as

$$
\begin{aligned}
(-\Delta)_{g}^{s} u-\gamma^{(s, g, \alpha)} \tilde{g}(u)= & \left(b^{(s, g, \alpha)}-\gamma^{(s, g, \alpha)}\right) \tilde{g}(u)^{+}-\left(a^{(s, g, \alpha)}-\gamma^{(s, g, \alpha)}\right) \tilde{g}(u)^{-} \\
& +\tau g\left(\left|\phi_{1}^{(s, g, \alpha)}\right|\right) \frac{\phi_{1}^{(s, g, \alpha)}}{\left|\phi_{1}^{(s, g, \alpha)}\right|}
\end{aligned}
$$

Then, (1.1) can be rewritten as

$$
\begin{aligned}
\left(I \circ \tilde{g} \circ D_{s} \circ \tilde{g}^{-1}-\gamma^{(s, g, \alpha)}\right) \tilde{g}(u)= & \left(b^{(s, g, \alpha)}-\gamma^{(s, g, \alpha)}\right) \tilde{g}(u)^{+}-\left(a^{(s, g, \alpha)}-\gamma^{(s, g, \alpha)}\right) \tilde{g}(u)^{-} \\
& +\tau g\left(\left|\phi_{1}^{(s, g, \alpha)}\right|\right) \frac{\phi_{1}^{(s, g, \alpha)}}{\left|\phi_{1}^{(s, g, \alpha)}\right|} .
\end{aligned}
$$

or equivalently

$$
\begin{aligned}
\tilde{g}(u)= & \left(I \circ \tilde{g} \circ D_{s} \circ \tilde{g}^{-1}-\gamma^{(s, g, \alpha)}\right)^{-1}\left(\left(b^{(s, g, \alpha)}-\gamma^{(s, g, \alpha)}\right) \tilde{g}(u)^{+}-\left(a^{(s, g, \alpha)}-\gamma^{(s, g, \alpha)}\right) \tilde{g}(u)^{-}\right. \\
& \left.+\tau g\left(\left|\phi_{1}^{(s, g, \alpha)}\right|\right) \frac{\phi_{1}^{(s, g, \alpha)}}{\left|\phi_{1}^{(s, g, \alpha)}\right|}\right) .
\end{aligned}
$$

The operator norm of the operator $\left(I \circ \tilde{g} \circ D_{s} \circ \tilde{g}^{-1}-\gamma^{(s, g, \alpha)}\right)^{-1}$ is

$$
\left\|\left(I \circ \tilde{g} \circ D_{s} \circ \tilde{g}^{-1}-\gamma^{(s, g, \alpha)}\right)^{-1}\right\|=\frac{1}{\min \left\{\gamma^{(s, g, \alpha)}-\lambda_{j}^{(s, g, \alpha)}, \lambda_{j+1}^{(s, g, \alpha)}-\gamma^{(s, g, \alpha)}\right\}} .
$$

Thus, we have that

$$
\begin{aligned}
\left(I \circ \tilde{g} \circ D_{s} \circ \tilde{g}^{-1}-\gamma^{(s, g, \alpha)}\right)^{-1}\left(\left(b^{(s, g, \alpha)}-\gamma^{(s, g, \alpha)}\right) \tilde{g}(u)^{+}-\left(a^{(s, g, \alpha)}-\gamma^{(s, g, \alpha)}\right) \tilde{g}(u)^{-}\right. \\
\left.\quad+\tau g\left(\left|\phi_{1}^{(s, g, \alpha)}\right|\right) \frac{\phi_{1}^{(s, g, \alpha)}}{\left|\phi_{1}^{(s, g, \alpha)}\right|}\right) \\
\leq \frac{1}{\min \left\{\gamma^{(s, g, \alpha)}-\lambda_{j}^{(s, g, \alpha)}, \lambda_{j+1}^{(s, g, \alpha)}-\gamma^{(s, g, \alpha)}\right\} \|\left(b^{(s, g, \alpha)}-\gamma^{(s, g, \alpha)}\right) \tilde{g}(u)^{+}} \\
\quad-\left(a^{(s, g, \alpha)}-\gamma^{(s, g, \alpha)}\right) \tilde{g}(u)^{-}+\tau g\left(\left|\phi_{1}^{(s, g, \alpha)}\right|\right) \frac{\phi_{1}^{(s, g, \alpha)}}{\left|\phi_{1}^{(s, g, \alpha)}\right|} \|_{G} .
\end{aligned}
$$

Let us set the right-hand side of (2.9) as

$$
\begin{aligned}
S^{(s, g, \alpha)}(\tilde{g}(u))= & \left(I \circ \tilde{g} \circ D_{s} \circ \tilde{g}^{-1}-\gamma^{(s, g, \alpha)}\right)^{-1}\left(\left(b^{(s, g, \alpha)}-\gamma^{(s, g, \alpha)}\right) \tilde{g}(u)^{+}\right. \\
& \left.-\left(a^{(s, g, \alpha)}-\gamma^{(s, g, \alpha)}\right) \tilde{g}(u)^{-}+\tau g\left(\left|\phi_{1}^{(s, g, \alpha)}\right|\right) \frac{\phi_{1}^{(s, g, \alpha)}}{\left|\phi_{1}^{(s, g, \alpha)}\right|}\right) .
\end{aligned}
$$


Then, $S^{(s, g, \alpha)}(\tilde{g})$ satisfies

$$
\begin{aligned}
\| & S^{(s, g, \alpha)}(\tilde{g}(u))-S^{(s, g, \alpha)}(\tilde{g}(v)) \|_{s, G} \\
= & \|\left(I \circ \tilde{g} \circ D_{s} \circ \tilde{g}^{-1}-\gamma^{(s, g, \alpha)}\right)^{-1}\left(\left(b^{(s, g, \alpha)}-\gamma^{(s, g, \alpha)}\right) \tilde{g}(u)^{+}-\left(a^{(s, g, \alpha)}-\gamma^{(s, g, \alpha)}\right) \tilde{g}(u)^{-}\right) \\
& -\left(I \circ \tilde{g} \circ D_{s} \circ \tilde{g}^{-1}-\gamma^{(s, g, \alpha)}\right)^{-1}\left(\left(b^{(s, g, \alpha)}-\gamma^{(s, g, \alpha)}\right) \tilde{g}(v)^{+}-\left(a^{(s, g, \alpha)}-\gamma^{(s, g, \alpha)}\right) \tilde{g}(u)^{-}\right) \|_{s, G} \\
\leq & \frac{1}{\min \left\{\gamma^{(s, g, \alpha)}-\lambda_{j}^{(s, g, \alpha)}, \lambda_{j+1}^{(s, g, \alpha)}-\gamma^{(s, g, \alpha)}\right\}} \|\left(\left(b^{(s, g, \alpha)}-\gamma^{(s, g, \alpha)}\right) \tilde{g}(u)^{+}\right. \\
& \left.-\left(a^{(s, g, \alpha)}-\gamma^{(s, g, \alpha)}\right) \tilde{g}(u)^{-}\right)-\left(\left(b^{(s, g, \alpha)}-\gamma^{(s, g, \alpha)}\right) \tilde{g}(v)^{+}-\left(a^{(s, g, \alpha)}-\gamma^{(s, g, \alpha)}\right) \tilde{g}(v)^{-}\right) \|_{G} \\
\leq & \frac{1}{\min \left\{\gamma^{(s, g, \alpha)}-\lambda_{j}^{(s, g, \alpha)}, \lambda_{j+1}^{(s, g, \alpha)}-\gamma^{(s, g, \alpha)}\right\}} \frac{b^{s, g, \alpha}-a^{(s, g, \alpha)}}{2}\|\tilde{g}(u)-\tilde{g}(v)\|_{G}
\end{aligned}
$$

Since $\frac{1}{\min \left\{\gamma^{(s, g, \alpha)}-\lambda_{j}^{(s, g, \alpha)}, \lambda_{j+1}^{(s, g, \alpha)}-\gamma^{(s, g, \alpha)}\right\}} \frac{b^{s, g, \alpha}-a^{(s, g, \alpha)}}{2}<1, S^{(s, g, \alpha)}$ is a contraction mapping with respect to the variable $\tilde{g}(u)=g(|u|) \frac{u}{|u|}$. Thus, (2.9) has a unique solution $\tilde{g}(u)$ for each $s, g$, $\alpha$. Since $\tilde{g}$ is a strictly increasing continuous function, there exists a unique $u$ for a unique given value $\tilde{g}(u)$. Thus, $(1.1)$ has a unique solution $u \neq 0$.

\section{Proof of Theorem 1.2}

(i) We assume that $a^{(s, g, \alpha)}<b^{(s, g, \alpha)},-\infty<a^{(s, g, \alpha)}<\lambda_{1}^{(s, g, \alpha)}<b^{(s, g, \alpha)}<\lambda_{2}^{(s, g, \alpha)}, \tau>0$. Then, (1.1) can be rewritten as

$$
\begin{aligned}
(-\Delta)_{g}^{s} u-\lambda_{1}^{(s, g, \alpha)} g(|u|) \frac{u}{|u|}= & \left(b^{(s, g, \alpha)}-\lambda_{1}^{(s, g, \alpha)}\right) g(|u|) \frac{u^{+}}{|u|}-\left(a^{(s, g, \alpha)}-\lambda_{1}^{(s, g, \alpha)} g(|u|) \frac{u^{-}}{|u|}\right. \\
& +\tau g\left(\left|\phi_{1}^{(s, g, \alpha)}\right|\right) \frac{\phi_{1}^{(s, g, \alpha)}}{\left|\phi_{1}^{(s, g, \alpha)}\right|}
\end{aligned}
$$

Taking the inner product with $\phi_{1}^{(s, g, \alpha)}>0$ in (3.1), we have

$$
\begin{aligned}
& \left\langle(-\Delta)_{g}^{s} u-\lambda_{1}^{(s, g, \alpha)} g(|u|) \frac{u}{|u|}, \phi_{1}^{(s, g, \alpha)}\right\rangle \\
& =\left\langle\left(b^{(s, g, \alpha)}-\lambda_{1}^{(s, g, \alpha)}\right) g(|u|) \frac{u^{+}}{|u|}-\left(a^{(s, g, \alpha)}-\lambda_{1}^{(s, g, \alpha)} g(|u|) \frac{u^{-}}{|u|}\right.\right. \\
& \left.\quad+\tau g\left(\left|\phi_{1}^{(s, g, \alpha)}\right|\right) \frac{\phi_{1}^{(s, g, \alpha)}}{\left|\phi_{1}^{(s, g, \alpha)}\right|}, \phi_{1}^{(s, g, \alpha)}\right\rangle .
\end{aligned}
$$

The left-hand side of (3.2) is equal to 0. On the other hand, the right-hand side of (3.2) is positive because $b^{(s, g, \alpha)}-\lambda_{1}^{(s, g, \alpha)}>0,-\left(a^{(s, g, \alpha)}-\lambda_{1}^{(s, g, \alpha)}\right)>0$ and $\tau g\left(\left|\phi_{1}^{(s, g, \alpha)}\right|\right) \frac{\phi_{1}^{(s, g, \alpha)}}{\left|\phi_{1}^{(s, g, \alpha)}\right|}>0$ for $\tau>0$ and $\phi_{1}^{(s, g, \alpha)}>0$. Thus, if $\tau>0$, then there is no solution for (1.1).

(ii) We assume that $\tau=0$. Then, (3.2) is reduced to the equation

$$
\begin{aligned}
& \left\langle(-\Delta)_{g}^{s} u-\lambda_{1}^{(s, g, \alpha)} g(|u|) \frac{u}{|u|}, \phi_{1}^{(s, g, \alpha)}\right\rangle \\
& =\left\langle\left(b^{(s, g, \alpha)}-\lambda_{1}^{(s, g, \alpha)}\right) g(|u|) \frac{u^{+}}{|u|}-\left(a^{(s, g, \alpha)}-\lambda_{1}^{(s, g, \alpha)} g(|u|) \frac{u^{-}}{|u|}, \phi_{1}^{(s, g, \alpha)}\right\rangle .\right.
\end{aligned}
$$


i.e.,

$$
\begin{aligned}
& \left\langle(-\Delta)_{g}^{s} u-\lambda_{1}^{(s, g, \alpha)} g(|u|) \frac{u}{|u|}, \phi_{1}^{(s, g, \alpha)}\right\rangle \\
& \quad=0=\int_{\Omega}\left[\left(\left(b^{(s, g, \alpha)}-\lambda_{1}^{(s, g, \alpha)}\right) g(|u|) \frac{u^{+}}{|u|}-\left(a^{(s, g, \alpha)}-\lambda_{1}^{(s, g, \alpha)}\right) g(|u|) \frac{u^{-}}{|u|}\right) \phi_{1}^{(s, g, \alpha)}\right] d x .
\end{aligned}
$$

Since $b^{(s, g, \alpha)}-\lambda_{1}^{(s, g, \alpha)}>0$ and $-\left(a^{(s, g, \alpha)}-\lambda_{1}^{(s, g, \alpha)}\right)>0$, the only possibility to hold (3.4) is that $u=0$ in $W_{0}^{s} L_{G}(\Omega) \cap C(\Omega)$.

(iii) We assume that $a^{(s, g, \alpha)}<b^{(s, g, \alpha)},-\infty<a^{(s, g, \alpha)}<\lambda_{1}^{(s, g, \alpha)}<b^{(s, g, \alpha)}<\lambda_{2}^{(s, g, \alpha)}, \tau<0$. Let $V$ be a subspace of $L_{G}(\Omega)$ spanned by $\phi_{1}^{(s, g, \alpha)}$ and $W$ be the orthogonal complement of $V$ in $L_{G}(\Omega)$. Then,

$$
L_{G}(\Omega)=V \oplus W
$$

Let $Q$ be an orthogonal projection in $L_{G}(\Omega)$ onto $V$ and $I-Q$ be the orthogonal projection onto $W$. Then,

$$
Q u=\left(\int_{\Omega} u \phi_{1}^{(s, g, \alpha)}\right) \phi_{1}^{(s, g, \alpha)} \quad \text { for all } u \in L_{G}(\Omega) .
$$

Let $u \in L_{G}(\Omega)$. Then, $u$ can be written as

$$
u=v+z, \quad v=Q u, \quad w=(I-Q) z .
$$

We note that $Q$ commutes with $D=\frac{d}{d x}$. Then, (1.1) is equivalent to a pair of equations

$$
\begin{aligned}
& Q(-\Delta)_{g}^{s}(v+z)=Q\left(b^{(s, g, \alpha)} g(|v+z|) \frac{(v+z)^{+}}{|v+z|}-a^{(s, g, \alpha)} g(|(v+z)|) \frac{(v+z)^{-}}{|v+z|}\right. \\
& \left.\quad+\tau g\left(\left|\phi_{1}^{(s, g, \alpha)}\right|\right) \frac{\phi_{1}^{(s, g, \alpha)}}{\left|\phi_{1}^{(s, g, \alpha)}\right|}\right), \\
& (I-Q)(-\Delta)_{g}^{s}(v+z) \\
& =(I-Q)\left(b^{(s, g, \alpha)} g(|v+z|) \frac{(v+z)^{+}}{|v+z|}-a^{(s, g, \alpha)} g(|v+z|) \frac{(v+z)^{-}}{|v+z|}\right) .
\end{aligned}
$$

Since $(-\Delta)_{g}^{s} u=\left(I \circ \tilde{g} \circ D_{s} \circ \tilde{g}^{-1}\right)(\tilde{g}(u))$, (3.6) can be rewritten as

$$
(I-Q)(\tilde{g}(v+z))=\left(I \circ \tilde{g} \circ D_{s} \circ \tilde{g}^{-1}\right)^{-1}(I-Q)\left(b^{(s, g, \alpha)} \tilde{g}(v+z)^{+}-a^{(s, g, \alpha)} \tilde{g}(v+z)^{-}\right) .
$$

The operator norm of the operator $\left(I \circ \tilde{g} \circ D_{s} \circ \tilde{g}^{-1}\right)^{-1}(I-Q)$ is

$$
\left\|\left(I \circ \tilde{g} \circ D_{s} \circ \tilde{g}^{-1}\right)^{-1}(I-Q)\right\|=\frac{1}{\lambda_{2}^{(s, g, \alpha)}} .
$$

We claim that for fixed $v \in V$, (3.6) has a unique solution $z(v)$. We first shall show that there exists a unique $g(|v+z|) \frac{v+z}{|v+z|}$ satisfying (3.6) for any given $v \in V$ and some $z \in W$. In 
fact, we suppose that there exist two $z_{1}(v)$ and $z_{1}(v)$ satisfying (3.6) for fixed $v \in V$. Since $g$ is a strictly increasing function, there exist two $\tilde{g}\left(v+z_{1}\right)=g\left(\left|v+z_{1}\right|\right) \frac{v+z_{1}}{\left|v+z_{1}\right|}$ and $\tilde{g}\left(v+z_{2}\right)=$ $g\left(\left|v+z_{2}\right|\right) \frac{v+z_{2}}{\left|v+z_{2}\right|}$ satisfying (3.6) for the given two $z_{1}(v)$ and $z_{1}(v)$. Then we have, from (3.7),

$$
\begin{aligned}
(I-Q) & \left(\tilde{g}\left(v+z_{1}\right)-\tilde{g}\left(v+z_{2}\right)\right) \\
= & \left(I \circ \tilde{g} \circ D_{s} \circ \tilde{g}^{-1}\right)^{-1}(I-Q)\left(b^{(s, g, \alpha)} \tilde{g}\left(v+z_{1}\right)^{+}-a^{(s, g, \alpha)} \tilde{g}\left(v+z_{1}\right)^{-}\right) \\
& \quad-\left(I \circ \tilde{g} \circ D_{s} \circ \tilde{g}^{-1}\right)^{-1}(I-Q)\left(b^{(s, g, \alpha)} \tilde{g}\left(v+z_{2}\right)^{+}-a^{(s, g, \alpha)} \tilde{g}\left(v+z_{2}\right)^{-}\right) .
\end{aligned}
$$

Taking the inner product of (3.8) with $\tilde{g}\left(v+z_{1}\right)-\tilde{g}\left(v+z_{2}\right)$, we have

$$
\begin{aligned}
(I-Q) & \left(\tilde{g}\left(v+z_{1}\right)-\tilde{g}\left(v+z_{2}\right)\right)^{2} \\
= & {\left[\left(I \circ \tilde{g} \circ D_{s} \circ \tilde{g}^{-1}\right)^{-1}(I-Q)\left(b^{(s, g, \alpha)} \tilde{g}\left(v+z_{1}\right)^{+}-a^{(s, g, \alpha)} \tilde{g}\left(v+z_{1}\right)^{-}\right)\right.} \\
& \left.\quad-\left(I \circ \tilde{g} \circ D_{s} \circ \tilde{g}^{-1}\right)^{-1}(I-Q)\left(b^{(s, g, \alpha)} \tilde{g}\left(v+z_{2}\right)^{+}-a^{(s, g, \alpha)} \tilde{g}\left(v+z_{2}\right)^{-}\right)\right] \\
& \cdot\left(\tilde{g}\left(v+z_{1}\right)-\tilde{g}\left(v+z_{2}\right)\right) .
\end{aligned}
$$

Then, the right-hand side of (3.9) is equal to

$$
\begin{aligned}
& {\left[\left(I \circ \tilde{g} \circ D_{s} \circ \tilde{g}^{-1}\right)^{-1}(I-Q)\left(b^{(s, g, \alpha)} \tilde{g}\left(v+z_{1}\right)^{+}-a^{(s, g, \alpha)} \tilde{g}\left(v+z_{1}\right)^{-}\right)\right.} \\
& \left.\quad-\left(I \circ \tilde{g} \circ D_{s} \circ \tilde{g}^{-1}\right)^{-1}(I-Q)\left(b^{(s, g, \alpha)} \tilde{g}\left(v+z_{2}\right)^{+}-a^{(s, g, \alpha)} \tilde{g}\left(v+z_{2}\right)^{-}\right)\right] \\
& \quad \times\left(\tilde{g}\left(v+z_{1}\right)-\tilde{g}\left(v+z_{2}\right)\right) \\
& \leq \leq \frac{b^{(s, g, \alpha)}}{\lambda_{2}^{(s, g, \alpha)}}\left(\tilde{g}\left(v+z_{1}\right)-\tilde{g}\left(v+z_{2}\right)\right)^{2},
\end{aligned}
$$

which is a contradiction because $\frac{b^{(s, g, \alpha)}}{\lambda_{2}^{(s, g, \alpha)}}<1$. Thus, $\tilde{g}\left(v+z_{1}\right)=\tilde{g}\left(v+z_{2}\right)$. Since $\tilde{g}$ is a strictly increasing continuous function, $z_{1}=z_{2}$ for fixed $v \in V$. Thus, there exists a unique $z(v) \in$ $W$ for any given $v \in V$. We note that $z=0$ is a solution of (3.6) for every $v \in V, v>0$ or $v<0$ everywhere in $\Omega$. If $v>0$ and $z=0$, then

$$
(I-Q)(-\Delta)_{g}^{s}(v)=(I-Q)\left(b^{(s, g, \alpha)} g(|v|) \frac{v^{+}}{|v|}-a^{(s, g, \alpha)} g(|v|) \frac{v^{-}}{|v|}\right)=0 .
$$

If $v<0$ and $z=0$, then $(I-Q)\left(b^{(s, g, \alpha)} g(|v|) \frac{v^{+}}{|v|}-a^{(s, g, \alpha)} g(|v|) \frac{v^{-}}{|v|}\right)=0$. Thus, (1.1) is reduced to

$$
Q(-\Delta)_{g}^{s}(v)=Q\left(b^{(s, g, \alpha)} g(|v|) \frac{\nu^{+}}{|v|}-a^{(s, g, \alpha)} g(|v|) \frac{v^{-}}{|v|}+\tau g\left(\left|\phi_{1}^{(s, g, \alpha)}\right|\right) \frac{\phi_{1}^{(s, g, \alpha)}}{\left|\phi_{1}^{(s, g, \alpha)}\right|}\right),
$$

where $v=c^{(s, g, \alpha)} \phi_{1}^{(s, g, \alpha)}, c \in R$.

If $c^{(s, g, \alpha)}>0$ and $\tau<0$, then

$$
\begin{aligned}
& \lambda_{1}^{(s, g, \alpha)} g\left(c^{(s, g, \alpha)} \phi_{1}^{(s, g, \alpha)}\right) \frac{c^{(s, g, g)} \phi_{1}^{(s, g, \alpha)}}{c^{(s, g, \alpha)} \phi_{1}^{(s, g, \alpha)}} \\
& \quad=b^{(s, g, \alpha)} g\left(c^{(s, g, \alpha)} \phi_{1}^{(s, g, \alpha)}\right) \frac{c^{(s, g, \alpha)} \phi_{1}^{(s, g, \alpha)}}{c^{(s, g, \alpha)} \phi_{1}^{(s, g, \alpha)}}+\tau g\left(\left|\phi_{1}^{(s, g, \alpha)}\right|\right) \frac{\phi_{1}^{(s, g, \alpha)}}{\left|\phi_{1}^{(s, g, \alpha)}\right|} .
\end{aligned}
$$


Thus, we have

$$
c^{(s, g, \alpha)}=\frac{g^{-1}\left(\frac{\tau}{\lambda_{1}^{(s, g, \alpha)}-b^{(s, g, \alpha)}} g\left(\left|\phi_{1}^{(s, g, \alpha)}\right|\right)\right)}{\phi_{1}^{(s, g, \alpha)}}
$$

If $c^{(s, g, \alpha)}<0$ and $\tau<0$, then

$$
\begin{gathered}
\lambda_{1}^{(s, g, \alpha)} g\left(-c^{(s, g, \alpha)} \phi_{1}^{(s, g, \alpha)}\right) \frac{c^{(s, g, \alpha)} \phi_{1}^{(s, g, \alpha)}}{-c^{(s, g, \alpha)} \phi_{1}^{(s, g, \alpha)}} \\
\quad=-a^{(s, g, \alpha)} g\left(-c^{(s, g, \alpha)} \phi_{1}^{(s, g, \alpha)}\right) \frac{-c \phi_{1}^{(s, g, \alpha)}}{-c \phi_{1}^{(s, g, \alpha)}}+\tau g\left(\left|\phi_{1}^{(s, g, \alpha)}\right|\right) \frac{\phi_{1}^{(s, g, \alpha)}}{\left|\phi_{1}^{(s, g, \alpha)}\right|} .
\end{gathered}
$$

Thus, we have

$$
c^{(s, g, \alpha)}=-\frac{g^{-1}\left(\frac{\tau}{a^{(s, g, \alpha)}-\lambda_{1}^{(s, g, \alpha)}} g\left(\left|\phi_{1}^{(s, g, \alpha)}\right|\right)\right)}{\phi_{1}^{(s, g, \alpha)}} .
$$

Thus, (1.1) has exactly two solutions in $W_{0}^{s} L_{G}(\Omega) \cap C(\Omega)$.

\section{Proofs of Theorem 1.3 (i), (ii) and (iii)}

The proofs of Theorem 1.3 (i) and (ii) are the same as those of Theorem 1.2 (i) and (ii).

For the proof of Theorem 1.3 (iii), we assume that $0<s<1, a^{(s, g, \alpha)}<b^{(s, g, \alpha)},-\infty<$ $a^{(s, g, \alpha)}<\lambda_{1}^{(s, g, \alpha)}, \lambda_{2}^{(s, g, \alpha)}<b^{(s, g, \alpha)}<\lambda_{3}^{(s, g, \alpha)}, \tau<0$ and $u \in W_{0}^{s} L_{G}(\Omega) \cap C(\Omega)$. We also assume that the conditions (1.6)-(1.9) hold. We note that (1.1) has a positive solution $g^{-1}\left(\frac{\tau}{\lambda_{1}^{(s, g, \alpha)}-b^{(s, g, \alpha)}} g\left(\left|\phi_{1}^{(s, g, \alpha)}\right|\right) \frac{\phi_{1}^{(s, g, \alpha)}}{\left|\phi_{1}^{(s, g, \alpha)}\right|}\right)>0$ in $W_{0}^{s} L_{G}(\Omega) \cap C(\Omega)$ and a negative solution $-g^{-1}\left(\frac{\tau}{a^{(s, g, \alpha)}-\lambda_{1}^{(s, g, \alpha)}} g\left(\left|\phi_{1}^{(s, g, \alpha)}\right|\right) \frac{\phi_{1}^{(s, g, \alpha)}}{\left|\phi_{1}^{(s, g, \alpha)}\right|}\right)<0$ in $W_{0}^{s} L_{G}(\Omega) \cap C(\Omega)$.

Lemma 4.1 (A priori bound) Assume that $0<s<1,-\infty<a^{(s, g, \alpha)}<\lambda_{1}^{(s, g, \alpha)}, \lambda_{2}^{(s, g, \alpha)}<b^{(s, g, \alpha)}<$ $\lambda_{3}^{(s, g, \alpha)}$ and $\tau \in R$. Then, there exist $\tau_{1}^{(s, g, \alpha)}<0, \tau_{2}^{(s, g, \alpha)}>0$, a constant $C^{(s, g, \alpha))}>0$ and a constant $C_{1}^{(s, g, \alpha))}>0$ depending only on $a^{(s, g, \alpha)}$ and $b^{(s, g, \alpha)}$ such that for any $\tau$ with $\tau_{1}^{(s, g, \alpha)} \leq \tau \leq$ $\tau_{2}^{(s, g, \alpha)}$, any solution $u$ of $(1.1)$ in $W_{0}^{s} L_{G}(\Omega) \cap C(\Omega)$ satisfies $\|u\|_{s, G}<C^{(s, g, \alpha)}$, and so satisfies $\|\tilde{g}(u)\|_{s, G}<C_{1}^{(s, g, \alpha)}$, where $\tilde{g}(u)=g(|u|) \frac{u}{|u|}$.

Proof Let $u$ be any solution of $(1.1)$ in $W_{0}^{s} L_{G}(\Omega) \cap C(\Omega)$. Suppose that any solution of (1.1) is not bounded. Then, there exists a sequence $\left(u_{n}\right)_{n}$ such that $\left\|u_{n}\right\|_{s, G} \rightarrow \infty$ so that

$$
(-\Delta)_{g}^{s} u_{n}=b^{(s, g, \alpha)} g\left(\left|u_{n}\right|\right) \frac{u_{n}^{+}}{\left|u_{n}\right|}-a^{(s, g, \alpha)} g\left(\left|u_{n}\right|\right) \frac{u_{n}^{-}}{\left|u_{n}\right|}+\tau g\left(\left|\phi_{1}^{(s, g, \alpha)}\right|\right) \frac{\phi_{1}^{(s, g, \alpha)}}{\left|\phi_{1}^{(s, g, \alpha)}\right|}
$$

or equivalently

$$
\tilde{g}\left(u_{n}\right)=\left(\tilde{g} \circ\left((-\Delta)_{g}^{s}\right)^{-1}\right)\left(b^{(s, g, \alpha)} \tilde{g}\left(u_{n}\right)^{+}-a^{(s, g, \alpha)} \tilde{g}\left(u_{n}\right)^{=}+\tau g\left(\left|\phi_{1}^{(s, g, \alpha)}\right|\right) \frac{\phi_{1}^{(s, g, \alpha)}}{\left|\phi_{1}^{(s, g, \alpha)}\right|}\right) \quad \text { in } \Omega \text {. }
$$

Since $\left\|u_{n}\right\|_{s, G} \rightarrow \infty$ and $g$ is a strictly increasing continuous function, $g\left(\left|u_{n}\right|\right) \frac{u_{n}}{\left|u_{n}\right|} \rightarrow$ $\infty$. Let us consider $\left\{\frac{g\left(\left|u_{n}\right|\right) \frac{u_{n}}{\left|u_{n}\right|}}{\left\|g\left(\left|u_{n}\right|\right) \mid \frac{u_{n}}{\left|u_{n}\right|}\right\|_{s, G}}\right\}_{n}$. Since $\left\|\left(\frac{g\left(\left|u_{n}\right|\right) \frac{u_{n}}{\left|u_{n}\right|}}{\left\|g\left(\left|u_{n}\right|\right) \mid \frac{u_{n}}{\left|u_{n}\right|}\right\|_{s, G}}\right)_{n}\right\|_{s, G}=1,\left\{\frac{g\left(\left|u_{n}\right|\right) \frac{u_{n}}{\left|u_{n}\right|}}{\left\|g\left(\left|u_{n}\right|\right) \mid \frac{u_{n}}{\left|u_{n}\right|}\right\|_{s, G}}\right\}_{n}$ is 
bounded. Thus, there exists a subsequence, up to a subsequence, $\left\{\frac{g\left(\left|u_{n}\right|\right) \frac{u_{n}}{\mid u_{n} \|}}{\left\|g\left(\left|u_{n}\right|\right) \frac{u_{n}}{\left|u_{n}\right|}\right\|_{s, G}}\right\}_{n}$ such

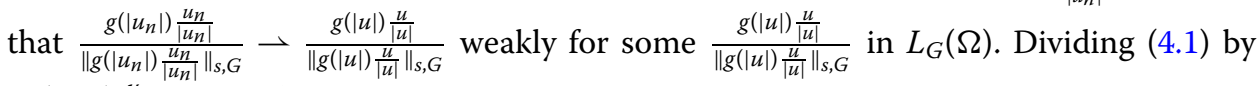
$\left\|g\left(\left|u_{n}\right|\right) \frac{u_{n}}{\left|u_{n}\right|}\right\|_{s, G}$, we have

$$
\begin{aligned}
\frac{(-\Delta)_{g}^{s} u_{n}}{\left\|g\left(\left|u_{n}\right|\right) \frac{u_{n}}{\left|u_{n}\right|}\right\|_{s, G}}= & b^{(s, g, \alpha)} \frac{g\left(\left|u_{n}\right|\right) \frac{u_{n}^{+}}{\left|u_{n}\right|}}{\left\|g\left(\left|u_{n}\right|\right) \frac{u_{n}}{\left|u_{n}\right|}\right\|_{s, G}}-a^{(s, g, \alpha)} \frac{g\left(\left|u_{n}\right|\right) \frac{u_{n}^{-}}{\left|u_{n}\right|}}{\left\|g\left(\left|u_{n}\right|\right) \frac{u_{n}}{\left|u_{n}\right|}\right\|_{s, G}} \\
& +\frac{\tau g\left(\left|\phi_{1}^{(s, g, \alpha)}\right|\right) \frac{\phi_{1}^{(s, g, \alpha)}}{\left|\phi_{1}^{(s, g, \alpha)}\right|}}{\left\|g\left(\left|u_{n}\right|\right) \frac{u_{n}}{\left|u_{n}\right|}\right\|_{s, G}} \text { in } \Omega .
\end{aligned}
$$

Since, by Lemma 2.1, the embedding $W_{0}^{s} L_{G}(\Omega) \hookrightarrow L_{G}(\Omega)$ is compact, and $\left((-\Delta)_{g}^{s}\right)^{-1}$ is a compact operator, $\frac{g\left(\left|u_{n}\right|\right) \frac{u_{n}}{\left|u_{n}\right|}}{\| g\left(\left|u_{n}\right|\right)} \rightarrow \frac{g(|u|) \frac{u}{|u|}}{\| u_{n} \mid} \|_{s, G}$ strongly in $W_{0}^{s} L_{G}(\Omega)$. Limiting (4.2) as $n \rightarrow$ $\infty$, we have

$$
\frac{(-\Delta)_{g}^{s} u}{\left\|g(|u|) \frac{u}{|u|}\right\|_{s, G}}=b^{(s, g, \alpha)} \frac{g(|u|) \frac{u^{+}}{|u|}}{\left\|g(|u|) \frac{u}{|u|}\right\|_{s, G}}-a^{(s, g, \alpha)} \frac{g(|u|) \frac{u^{-}}{|u|}}{\left\|g(|u|) \frac{u}{|u|}\right\|_{s, G}} \quad \text { in } \Omega .
$$

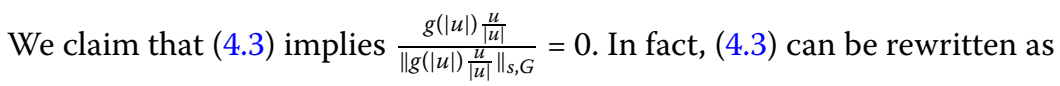

$$
\begin{aligned}
& \frac{(-\Delta)_{g}^{s} u}{\left\|g(|u|) \frac{u}{|u|}\right\|_{s, G}}-\lambda_{1}^{(s, g, \alpha)} \frac{g(|u|) \frac{u}{|u|}}{\left\|g(|u|) \frac{u}{|u|}\right\|_{s, G}} \\
& \quad=\left(b^{(s, g, \alpha)}-\lambda_{1}^{(s, g, \alpha)}\right) \frac{g(|u|) \frac{u^{+}}{|u|}}{\left\|g(|u|) \frac{u}{|u|}\right\|_{s, G}}-\left(a^{(s, g, \alpha)}-\lambda_{1}^{(s, g, \alpha)}\right) \frac{g(|u|) \frac{u^{-}}{|u|}}{\left\|g(|u|) \frac{u}{|u|}\right\|_{s, G}} \quad \text { in } \Omega .
\end{aligned}
$$

Taking the inner product with $\phi_{1}^{(s, g, \alpha)}>0$ on both sides of (4.4), we have

$$
\begin{aligned}
& \left\langle\frac{(-\Delta)_{g}^{s} u}{\left\|g(|u|) \frac{u}{|u|}\right\|_{s, G}}-\lambda_{1}^{(s, g, \alpha)} \frac{g(|u|) \frac{u}{|u|}}{\left\|g(|u|) \frac{u}{|u|}\right\|_{s, G}}, \phi_{1}^{(s, g, \alpha)}\right\rangle \\
& \quad=\left\langle\left(b^{(s, g, \alpha)}-\lambda_{1}^{(s, g, \alpha)}\right) \frac{g(|u|) \frac{u^{+}}{|u|}}{\left\|g(|u|) \frac{u}{|u|}\right\|_{s, G}}-\left(a^{(s, g, \alpha)}-\lambda_{1}^{(s, g, \alpha)}\right) \frac{g(|u|) \frac{u^{-}}{|u|}}{\left\|g(|u|) \frac{u}{|u|}\right\|_{s, G}}, \phi_{1}^{(s, g, \alpha)}\right\rangle \text { in } \Omega .
\end{aligned}
$$

The left-hand side of (4.5) is equal to 0 . On the other hand, the right-hand side of (4.5) is positive because $b^{(s, g, \alpha)}-\lambda_{1}^{(s, g, \alpha)}>0,-\left(a^{(s, g, \alpha)}-\lambda_{1}^{(s, g, \alpha)}\right)>0$ and $\phi_{1}^{(s, g, \alpha)}>0$. The only possibility

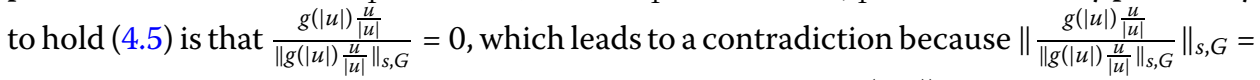
$1 \neq 0$. Thus, there exist a constant $C^{(s, g, \alpha))}>0$ and a constant $C_{1}^{(s, g, \alpha))}>0$ depending only on $a^{(s, g, \alpha)}$ and $b^{(s, g, \alpha)}$ such that any solution $u$ of (1.1) satisfies $\|u\|_{s, G}<C^{(s, g, \alpha)}$, and so satisfies $\|\tilde{g}(u)\|_{s, G}<C_{1}^{(s, g, \alpha)}$ since $g$ is a continuous function. Thus, the lemma is proved.

We shall consider the Leray-Schauder degree on a large ball.

Lemma 4.2 (Leray-Schauder degree on a large ball) Assume that $-\infty<a^{(s, g, \alpha)}<\lambda_{1}^{(s, g, \alpha)}$, $\lambda_{2}^{(s, g, \alpha)}<b^{(s, g, \alpha)}<\lambda_{3}^{(s, g, \alpha)}$. Then, there exist a constant $R^{(s, g, \alpha)}>0$ depending on $a^{(s, g, \alpha)}, b^{(s, g, \alpha)}$, 
$\tau, \tau_{1}^{(s, g, \alpha)}<0$ and $\tau_{2}^{(s, g, \alpha)}>0$ such that for any $\tau$ with $\tau_{1}^{(s, g, \alpha)} \leq \tau \leq \tau_{2}^{(s, g, \alpha)}$, the Leray-Schauder degree

$$
\begin{aligned}
& d_{L S}\left(\tilde{g}(u)-\left(\tilde{g} \circ\left((-\Delta)_{g}^{s}\right)^{-1}\right)\left(b^{(s, g, \alpha)} g(|u|) \frac{u^{+}}{|u|}-a^{(s, g, \alpha)} g(|u|) \frac{u^{-}}{|u|}\right.\right. \\
& \left.\left.\quad+\tau g\left(\left|\phi_{1}^{(s, g, \alpha)}\right|\right) \frac{\phi_{1}^{(s, g, \alpha)}}{\left|\phi_{1}^{(s, g, \alpha)}\right|}\right), B_{R^{(s, g, \alpha)}}(0), 0\right)=0 .
\end{aligned}
$$

Proof (1.1) can be rewritten as

$$
u-\left((-\Delta)_{g}^{s}\right)^{-1}\left(b^{(s, g, \alpha)} g(|u|) \frac{u^{+}}{|u|}-a^{(s, g, \alpha)} g(|u|) \frac{u^{-}}{|u|}+\tau g\left(\left|\phi_{1}^{(s, g, \alpha)}\right|\right) \frac{\phi_{1}^{(s, g, \alpha)}}{\left|\phi_{1}^{(s, g, \alpha)}\right|}\right)=0
$$

or equivalently

$$
\tilde{g}(u)-\left(\tilde{g} \circ\left((-\Delta)_{g}^{s}\right)^{-1}\right)\left(b^{(s, g, \alpha)} g(|u|) \frac{u^{+}}{|u|}-a^{(s, g, \alpha)} g(|u|) \frac{u^{-}}{|u|}+\tau g\left(\left|\phi_{1}^{(s, g, \alpha)}\right|\right) \frac{\phi_{1}^{(s, g, \alpha)}}{\left|\phi_{1}^{(s, g, \alpha)}\right|}\right)=0
$$

in $W_{g}^{s} L_{G}(\Omega) \cap C(\Omega)$.

Let us consider the homotopy

$$
\begin{aligned}
H^{(s, g, \alpha)}(x, \tilde{g}(u))= & \tilde{g}(u)-\left(\tilde{g} \circ\left((-\Delta)_{g}^{s}\right)^{-1}\right)\left(b^{(s, g, \alpha)} g(|u|) \frac{u^{+}}{|u|}-a^{(s, g, \alpha)} g(|u|) \frac{u^{-}}{|u|}\right. \\
& \left.+\tau g\left(\left|\phi_{1}^{(s, g, \alpha)}\right|\right) \frac{\phi_{1}^{(s, g, \alpha)}}{\left|\phi_{1}^{(s, g, \alpha)}\right|}\right) .
\end{aligned}
$$

By Theorem 1.3 (ii), for any $\tau>0,(4.6)$ has no solution. Thus, there exist $\tau_{2}^{(s, g, \alpha)}>0$ and a large $R^{(s, g, \alpha)}>0$ such that (4.7) has no zero in $B_{R^{(s, g, \alpha)}}(0)$ for any $\tau \geq \tau_{2}^{(s, g, \alpha)}$, and by a priori bound in Lemma 4.1, there exist $R^{(s, g, \alpha)}>0$ and $\tau_{1}^{(s, g, \alpha)}<0$ such that for any $\tau$ with $\tau_{1}^{(s, g, \alpha)} \leq$ $\tau \leq \tau_{2}^{(s, g, \alpha)}$, all solutions $u$ of (4.6) in $W_{0}^{s} L_{G}(\Omega) \cap C(\Omega)$ satisfy $\|\tilde{g}(u)\|_{s, G}<R^{(s, g, \alpha)}$ and (4.7) has no zero on $\partial B_{R^{(s, g, \alpha)}}(0)$ for any $\tau$ with $\tau_{1}^{(s, g, \alpha)} \leq \tau \leq \tau_{2}^{(s, g, \alpha)}$. Since

$$
\begin{aligned}
& d_{L S}\left(\tilde{g}(u)-\left(\tilde{g} \circ\left((-\Delta)_{g}^{s}\right)^{-1}\right)\left(b^{(s, g, \alpha)} g(|u|) \frac{u^{+}}{|u|}\right.\right. \\
& \left.\left.\quad-a^{(s, g, \alpha)} g(|u|) \frac{u^{-}}{|u|}+\tau_{2}^{(s, g, \alpha)} g\left(\left|\phi_{1}^{(s, g, \alpha)}\right|\right) \frac{\phi_{1}^{(s, g, \alpha)}}{\left|\phi_{1}^{(s, g, \alpha)}\right|}\right), B_{R^{(s, g, \alpha)}}(0), 0\right)=0,
\end{aligned}
$$

by homotopy arguments, for any $\tau$ with $\tau_{1}^{(s, g, \alpha)} \leq \tau \leq \tau_{2}^{(s, g, \alpha)}$, we have

$$
\begin{aligned}
& d_{L S}\left(\tilde{g}(u)-\left(\tilde{g} \circ\left((-\Delta)_{g}^{s}\right)^{-1}\right)\left(b^{(s, g, \alpha)} g(|u|) \frac{u^{+}}{|u|}-a^{(s, g, \alpha)} g(|u|) \frac{u^{-}}{|u|}\right.\right. \\
& \left.\left.\quad+\tau g\left(\left|\phi_{1}^{(s, g, \alpha)}\right|\right) \frac{\phi_{1}^{(s, g, \alpha)}}{\left|\phi_{1}^{(s, g, \alpha)}\right|}\right), B_{R^{(s, g, \alpha)}}(0), 0\right) \\
& =d_{L S}\left(\tilde{g}(u)-\left(\tilde{g} \circ\left((-\Delta)_{g}^{s}\right)^{-1}\right)\left(b^{(s, g, \alpha)} g(|u|) \frac{u^{+}}{|u|}-a^{(s, g, \alpha)} g(|u|) \frac{u^{-}}{|u|}\right.\right.
\end{aligned}
$$




$$
\begin{aligned}
& \left.\left.\left.+\tau g\left(\left|\phi_{1}^{(s, g, \alpha)}\right|\right) \frac{\phi_{1}^{(s, g, \alpha)}}{\left|\phi_{1}^{(s, g, \alpha)}\right|}\right)+\eta^{(s, g, \alpha)}\left(\tau_{2}^{(s, g, \alpha)}-\tau\right) \mid g\left(\left|\phi_{1}^{(s, g, \alpha)}\right|\right) \frac{\phi_{1}^{(s, g, \alpha)}}{\left|\phi_{1}^{(s, g, \alpha)}\right|}\right), B_{R^{(s, g, g)}}(0), 0\right) \\
= & d_{L S}\left(\tilde{g}(u)-\left(\tilde{g} \circ\left((-\Delta)_{g}^{s}\right)^{-1}\right)\left(b^{(s, g, \alpha)} g(|u|) \frac{u^{+}}{|u|}-a^{(s, g, \alpha)} g(|u|) \frac{u^{-}}{|u|}\right.\right. \\
& \left.\left.+\tau_{2}^{(s, g, \alpha)} g\left(\left|\phi_{1}^{(s, g, \alpha)}\right|\right) \frac{\phi_{1}^{(s, g, \alpha)}}{\left|\phi_{1}^{(s, g, \alpha)}\right|}\right), B_{R^{(s, g, \alpha)}}(0), 0\right)=0
\end{aligned}
$$

for any $0 \leq \eta^{(s, g, \alpha)} \leq 1$. Thus, the lemma is proved.

Lemma 4.3 (Modulus of continuity) Let $F$ be a compact set in $L_{G}(\Omega)$. Let $\xi>0$. Then, there exists a modulus of continuity $\beta: R \rightarrow R$ depending only on $F$ and $\xi$ such that

$$
\left\|\mid\left(|\tau|-\frac{\xi}{\eta}\right)^{+}\right\|_{L_{G}(\Omega)} \leq \beta(\eta) \quad \text { for all } \tau \in F .
$$

It follows that

$$
\left\|\mid(\eta \tau+\xi)^{-}\right\|_{L_{G}(\Omega)} \leq \eta \beta(\eta)
$$

and

$$
\left\|\mid(\eta \tau-\xi)^{+}\right\|_{L_{G}(\Omega)} \leq \eta \beta(\eta) \quad \text { for all } \tau \in F
$$

Proof For any $\tau \in F$, let $\tau_{\eta}=\left(|\tau|-\frac{\xi}{\eta}\right)^{+}$. Since $0 \leq \tau_{\eta} \leq|\tau|$ and $\tau_{\eta}(x) \rightarrow 0$ as $\eta \rightarrow 0$, it follows that $\left\|\tau_{\eta}\right\|_{L_{G}(\Omega)} \rightarrow 0$ for all $\tau \in F$. We claim that for given $\epsilon>0$, there exists $\delta>0$ such that if $\tau \in F$, then $\left\|\tau_{\eta}\right\|_{L_{G}(\Omega)} \leq 2 \epsilon$ for all $\eta \in[0, \delta]$. Choose $\left\{\tau_{i}, i=1, \ldots, N\right\}$ as an $\epsilon$ net for $F$. Choose $\delta$ so that $\left\|\left(\tau_{i}\right)_{\delta}\right\|_{L_{G}(\Omega)}<\epsilon$ for $i=1, \ldots, N$. Then, for any $\tau \in F$, there exists $\tau_{k}, \beta$, $\|\beta\|_{L_{G}(\Omega)}<\epsilon$ such that $\tau=\tau_{k}+\beta$. Since $(u+v)^{+} \leq u^{+}+v^{+}$, we have $\left\|\tau_{\delta}\right\|_{L_{G}(\Omega)} \leq\left(\tau_{k}\right)_{\delta}+|\beta|$ and therefore $\left\|\tau_{\eta}\right\|_{L_{G}(\Omega)} \leq\left\|\tau_{\delta}\right\|_{L_{G}(\Omega)}+\|\beta\|_{L_{G}(\Omega)} \leq 2 \epsilon$.

Lemma 4.4 Assume that $-\infty<a^{(s, g, \alpha)}<\lambda_{1}^{(s, g, \alpha)}, \lambda_{2}^{(s, g, \alpha)}<b^{(s, g, \alpha)}<\lambda_{3}^{(s, g, \alpha)}$. Then, there exist a constant $\epsilon_{0}^{(s, g, \alpha)}>0$ depending on $a^{(s, g, \alpha)}, b^{(s, g, \alpha)}, \tau$ and $\tau_{1}^{(s, g, \alpha)}<0$ such that for any $\tau$ such that $\tau_{1}^{(s, g, \alpha)} \leq \tau<0$, the Leray-Schauder degree

$$
\begin{gathered}
d_{L S}\left(\tilde{g}(u)-\left(\tilde{g} \circ\left((-\Delta)_{g}^{s}\right)^{-1}\right)\left(b^{(s, g, \alpha)} g(|u|) \frac{u^{+}}{|u|}-a^{(s, g, \alpha)} g(|u|) \frac{u^{-}}{|u|}\right.\right. \\
\left.\left.+\tau g\left(\left|\phi_{1}^{(s, g, \alpha)}\right|\right) \frac{\phi_{1}^{(s, g, \alpha)}}{\left|\phi_{1}^{(s, g, \alpha)}\right|}\right), B_{|\tau| \epsilon_{0}^{(s, g, \alpha)}}\left(\tilde{g}\left(u_{1}\right)\right), 0\right)=1,
\end{gathered}
$$

where $u_{1}=g^{-1}\left(\frac{\tau}{\lambda_{1}^{(s, g, \alpha)}-b^{(s, g, \alpha)}} g\left(\left|\phi_{1}^{(s, g, \alpha)}\right|\right) \frac{\phi_{1}^{(s, g, \alpha)}}{\left|\phi_{1}^{(s, g, \alpha)}\right|}\right)>0$ is a positive solution of $(1.1)$ in $W_{0}^{s} L_{G}(\Omega) \cap$ $C(\Omega)$.

Proof (1.1) can be rewritten as

$$
\left((-\Delta)_{g}^{s}-b^{(s, g, \alpha)} \tilde{g}\right) u=b^{(s, g, \alpha)} \tilde{g}(u)^{+}-a^{(s, g, \alpha)} \tilde{g}(u)^{-}-b^{(s, g, \alpha)} \tilde{g}(u)+\tau g\left(\left|\phi_{1}^{(s, g, \alpha)}\right|\right) \frac{\phi_{1}^{(s, g, \alpha)}}{\left|\phi_{1}^{(s, g, \alpha)}\right|}
$$


or equivalently

$$
\begin{aligned}
\tilde{g}(u)= & \left(\tilde{g} \circ\left((-\Delta)_{g}^{s}-b^{(s, g, \alpha)} \tilde{g}\right)^{-1}\right) \\
& \times\left(b^{(s, g, \alpha)} \tilde{g}(u)^{+}-a^{(s, g, \alpha)} \tilde{g}(u)^{-}-b^{(s, g, \alpha)} \tilde{g}(u)+\tau g\left(\left|\phi_{1}^{(s, g, \alpha)}\right|\right) \frac{\phi_{1}^{(s, g, \alpha)}}{\left|\phi_{1}^{(s, g, \alpha)}\right|}\right),
\end{aligned}
$$

where $\tilde{g}(u)=g(|u|) \frac{u}{|u|}$. Let us set $N^{(s, g, \alpha)}=\tilde{g} \circ\left((-\Delta)_{g}^{s}-b^{(s, g, \alpha)} \tilde{g}\right)^{-1}$. Then, (1.1) can be rewritten equivalently as

$$
\tilde{g}(u)=N^{(s, g, \alpha)}\left(b^{(s, g, \alpha)} \tilde{g}(u)^{+}-a^{(s, g, \alpha)} \tilde{g}(u)^{-}-b^{(s, g, \alpha)} \tilde{g}(u)+\tau g\left(\left|\phi_{1}^{(s, g, \alpha)}\right|\right) \frac{\phi_{1}^{(s, g, \alpha)}}{\left|\phi_{1}^{(s, g, \alpha)}\right|}\right) .
$$

Since $\left((-\Delta)_{g}^{s}\right)^{-1}$ is a compact operator on $L_{G}(\Omega)$, the operator $N^{(s, g, \alpha)}$ is a compact operator on $L_{G}(\Omega)$ for each $s, g$ and $\alpha$. Let $B^{(s, g, \alpha)}=\overline{N^{(s, g, \alpha)}(\bar{D})}$ be a closed unit ball for some closed ball $D$ in $L_{G}(\Omega)$. Let us set $\gamma^{(s, g, \alpha)}=\min \left\{b^{(s, g, \alpha)}-\lambda_{2}^{(s, g, \alpha)}, \lambda_{3}^{(s, g, \alpha)}-b^{(s, g, \alpha)}\right\}$. We can observe that the operator norm of $N^{(s, g, \alpha)}$ is $\left\|N^{(s, g, \alpha)}\right\|=\frac{1}{\gamma^{(s, g, \alpha)}}$. Let $\beta^{(s, g, \alpha)}$ be the modulus of continuity of Lemma 4.3 corresponding to $B^{(s, g, \alpha)}$ and $\tilde{g}\left(\xi^{(s, g, \alpha)}\right)=N^{(s, g, \alpha)}\left(\tau g\left(\left|\phi_{1}^{(s, g, \alpha)}\right|\right) \frac{\phi_{1}^{(s, g, \alpha)}}{\left|\phi_{1}^{(s, g, \alpha)}\right|}\right)=$ $\frac{\tau}{\lambda_{1}^{(s, g, \alpha)}-b^{(s, g, \alpha)}} g\left(\left|\phi_{1}^{(s, g, \alpha)}\right|\right) \frac{\phi_{1}^{(s, g, \alpha)}}{\left|\phi_{1}^{(s, g, \alpha)}\right|}$. Let us choose $\epsilon_{0}^{(s, g, \alpha)}>0$ so that

$$
\beta^{(s, g, \alpha)}\left(|\tau| \epsilon_{0}^{(s, g, \alpha)} \frac{1}{\gamma}\left(b^{(s, g, \alpha)}-a^{(s, g, \alpha)}\right)\right) \leq \frac{\left.\gamma^{(s, g, \alpha)}\right)^{2}}{4\left(b^{(s, g, \alpha)}-a^{(s, g, \alpha)}\right)^{2}} .
$$

We note that

$$
\left\|b^{(s, g, \alpha)} \tilde{g}(u)^{+}-a^{(s, g, \alpha)} \tilde{g}(u)^{-}-b^{(s, g, \alpha)} \tilde{g}(u)\right\|_{L_{G}(\Omega)} \leq\left(b^{(s, g, \alpha)}-a^{(s, g, \alpha)}\right)\left\|\tilde{g}(u)^{-}\right\|_{L_{G}(\Omega)} .
$$

For $\tilde{g}(u) \in \frac{\tau}{\lambda_{1}^{(s, g, \alpha)}-b^{(s, g, g)}} g\left(\left|\phi_{1}^{(s, g, \alpha)}\right|\right) \frac{\phi_{1}^{(s, g, \alpha)}}{\left|\phi_{1}^{(s, g, \alpha)}\right|}+|\tau| \epsilon_{0}^{(s, g, \alpha)} w$ with $w \in \bar{B}$

$$
\begin{aligned}
\left\|\tilde{g}(u)^{-}\right\|_{L_{G}(\Omega)} & =\left\|\left(\frac{\tau}{\lambda_{1}^{(s, g, \alpha)}-b^{(s, g, \alpha)}} g\left(\left|\phi_{1}^{(s, g, \alpha)}\right|\right) \frac{\phi_{1}^{(s, g, \alpha)}}{\left|\phi_{1}^{(s, g, \alpha)}\right|}+|\tau| \epsilon_{0}^{(s, g, \alpha)} w\right)^{-}\right\|_{L_{G}(\Omega)} \\
& \leq\left\|\left(|\tau| \epsilon_{0}^{(s, g, \alpha)} w\right)^{-}\right\|_{L_{G}(\Omega)} \leq|\tau| \epsilon_{0}^{(s, g, \alpha)}
\end{aligned}
$$

since $\frac{\tau}{\lambda_{1}^{(s, g, \alpha)}-b^{(s, g, \alpha)}} g\left(\left|\phi_{1}^{(s, g, \alpha)}\right|\right) \frac{\phi_{1}^{(s, g, \alpha)}}{\left|\phi_{1}^{(s, g, \alpha)}\right|}>0$. Let us set $F^{(s, g, \alpha)}(\tilde{g}(u))=N^{(s, g, \alpha)}\left(b^{(s, g, \alpha)} \tilde{g}(u)^{+}-\right.$ $\left.a^{(s, g, \alpha)} \tilde{g}(u)^{-}-b^{(s, g, \alpha)} \tilde{g}(u)\right)+N^{(s, g, \alpha)}\left(\tau g\left(\left|\phi_{1}^{(s, g, \alpha)}\right|\right) \frac{\phi_{1}^{(s, g, \alpha)}}{\left|\phi_{1}^{(s, g, \alpha)}\right|}\right.$. Then, $\left.F^{(s, g, \alpha)}(\tilde{g} g)(u)\right)$ can be rewritten as

$$
\begin{aligned}
F^{(s, g, \alpha)}(\tilde{g}(u))= & \frac{\tau}{\lambda_{1}^{(s, g, \alpha)}-b^{(s, g, \alpha)}} g\left(\left|\phi_{1}^{(s, g, \alpha)}\right|\right) \frac{\phi_{1}^{(s, g, \alpha)}}{\left|\phi_{1}^{(s, g, \alpha)}\right|} \\
& +N^{(s, g, \alpha)}\left(\left(b^{(s, g, \alpha)}-a^{(s, g, \alpha)}\right)|\tau| \epsilon_{0}^{(s, g, \alpha)} w\right), \quad w \in B .
\end{aligned}
$$


If $u$ is a solution of (4.8), then $\tilde{g}(u)=F^{(s, g, \alpha)}(\tilde{g}(u))$ and by Lemma 4.3,

$$
\begin{aligned}
\left\|(\tilde{g}(u))^{-}\right\|_{L_{G}(\Omega)}= & \|\left(\frac{\tau}{\lambda_{1}^{(s, g, \alpha)}-b^{(s, g, \alpha)}} g\left(\left|\phi_{1}^{(s, g, \alpha)}\right|\right) \frac{\phi_{1}^{(s, g, \alpha)}}{\left|\phi_{1}^{(s, g, \alpha)}\right|}\right. \\
& \left.+N^{(s, g, \alpha)}\left(\left(b^{(s, g, \alpha)}-a^{(s, g, \alpha)}\right)|\tau| \epsilon_{0}^{(s, g, \alpha)} w\right)\right)^{-} \|_{L_{G}(\Omega)} \\
\leq & \left\|\left(N^{(s, g, \alpha)}\left(\left(b^{(s, g, \alpha)}-a^{(s, g, \alpha)}\right)|\tau| \epsilon_{0}^{(s, g, \alpha)} w\right)\right)^{-}\right\|_{L_{G}(\Omega)} \\
\leq & |\tau| \epsilon_{0}^{(s, g, \alpha)} \frac{1}{\gamma}\left(b^{(s, g, \alpha)}-a^{(s, g, \alpha)}\right) \beta\left(|\tau| \epsilon_{0}^{(s, g, \alpha)} \frac{1}{\gamma}\left(b^{(s, g, \alpha)}-a^{(s, g, \alpha)}\right)\right) \\
\leq & \frac{\gamma^{(s, g, \alpha)}|\tau| \epsilon_{0}^{(s, g, \alpha)}}{4\left(b^{(s, g, \alpha)}-a^{(s, g, \alpha)}\right)} .
\end{aligned}
$$

Thus, we have

$$
\begin{aligned}
& \left\|N^{(s, g, \alpha)}\left(b^{(s, g, \alpha)} \tilde{g}(u)^{+}-a^{(s, g, \alpha)} \tilde{g}(u)^{-}-b^{(s, g, \alpha)} \tilde{g}(u)\right)\right\|_{L_{G}(\Omega)} \\
& \leq\left\|N\left(\left(b^{(s, g, \alpha)}-a^{(s, g, \alpha)}\right) \tilde{g}(u)^{-}\right)\right\|_{L_{G}(\Omega)} \\
& \leq \frac{1}{\gamma^{(s, g, \alpha)}}\left(b^{(s, g, \alpha)}-a^{(s, g, \alpha)}\left\|\tilde{g}(u)^{-}\right\|_{L_{G}(\Omega)} \leq \frac{1}{4}|\tau| \epsilon_{0}^{(s, g, \alpha)} .\right.
\end{aligned}
$$

Thus, we have shown that for any solution $u$ of $(1.1), \tilde{g}(u) \in \frac{\tau}{\lambda_{1}^{(s, g, \alpha)}-b^{(s, g, \alpha)}} g\left(\left|\phi_{1}^{(s, g, \alpha)}\right|\right) \frac{\phi_{1}^{(s, g, \alpha)}}{\mid \phi_{1}^{(s, g, \alpha)}}+$ $|\tau| \epsilon_{0}^{(s, g, \alpha)} \bar{B}$ belong to $\tilde{g}(u) \in \frac{\tau}{\lambda_{1}^{(s, g, \alpha)}-b^{(s, g, \alpha)}} g\left(\left|\phi_{1}^{(s, g, \alpha)}\right|\right) \frac{\phi_{1}^{(s, g, \alpha)}}{\left|\phi_{1}^{(s, g, \alpha)}\right|}+\frac{1}{4}|\tau| \epsilon_{0}^{(s, g, \alpha)} \bar{B}$. This estimate holds if we replace $b^{(s, g, \alpha)} \tilde{g}(u)^{+}-a^{(s, g, \alpha)} \tilde{g}(u)^{-}-b^{(s, g, \alpha)} \tilde{g}(u)$ by $\lambda^{(s, g, \alpha)}\left(b^{(s, g, \alpha)} \tilde{g}(u)^{+}-a^{(s, g, \alpha)} \tilde{g}(u)^{-}-\right.$ $b^{(s, g, \alpha)} \tilde{g}(u)$ with $0 \leq \lambda^{(s, g, \alpha)} \leq 1$. Thus, the equation

$$
\begin{aligned}
\tilde{g}(u)=\left(\tilde{g} \circ\left((-\Delta)_{g}^{s}\right)^{-1}\right)\left(\tau g\left(\left|\phi_{1}^{(s, g, \alpha)}\right|\right) \frac{\phi_{1}^{(s, g, \alpha)}}{\left|\phi_{1}^{(s, g, \alpha)}\right|}+b^{(s, g, \alpha)} \tilde{g}(u)\right. \\
+\lambda^{(s, g, \alpha)}\left(b^{(s, g, \alpha)} \tilde{g}(u)^{+}-a^{(s, g, \alpha)} \tilde{g}(u)^{-}-b^{(s, g, \alpha)} \tilde{g}(u)\right)
\end{aligned}
$$

has no solution on the boundary of the ball $B_{|\tau| \epsilon_{0}^{(s, g, \alpha)}}\left(\frac{\tau}{\lambda_{1}^{(s, g, \alpha)}-b^{(s, g, \alpha)}} g\left(\left|\phi_{1}^{(s, g, \alpha)}\right|\right) \frac{\phi^{(s, g, \alpha)}}{\left|\phi_{1}^{(s, g, \alpha)}\right|}\right)$ for $0 \leq$ $\lambda^{(s, g, \alpha)} \leq 1$. By the homotopy invariance degree,

$$
\begin{gathered}
d_{L S}\left(\tilde{g}(u)-\left(\tilde{g} \circ\left((-\Delta)_{g}^{s}\right)^{-1}\right)\left(\tau g\left(\left|\phi_{1}^{(s, g, \alpha)}\right|\right) \frac{\phi_{1}^{(s, g, \alpha 0}}{\left|\phi_{1}^{(s, g, \alpha)}\right|}+b^{(s, g, \alpha)} \tilde{g}(u)\right.\right. \\
\left.+\lambda^{(s, g, \alpha)}\left(b^{(s, g, \alpha)} \tilde{g}(u)^{+}-a^{(s, g, \alpha)} \tilde{g}(u)^{-}-b^{(s, g, \alpha)} \tilde{g}(u)\right)\right), \\
\left.B_{|\tau| \epsilon_{0}^{(s, g, \alpha)}}\left(\frac{\tau}{\lambda_{1}^{(s, g, \alpha)}-b^{(s, g, \alpha)}} g\left(\left|\phi_{1}^{(s, g, \alpha)}\right|\right) \frac{\phi^{(s, g, \alpha)}}{\left|\phi_{1}^{(s, g, \alpha)}\right|}\right), 0\right)
\end{gathered}
$$

is defined for $0 \leq \lambda^{(s, g, \alpha)} \leq 1$ and is independent of $\lambda^{(s, g, \alpha)}$. For $\lambda^{(s, g, \alpha)}=0$,

$$
d_{L S}\left(\tilde{g}(u)-\left(\tilde{g} \circ\left((-\Delta)_{g}^{s}\right)^{-1}\right)\left(\tau g\left(\left|\phi_{1}^{(s, g, \alpha)}\right|\right) \frac{\phi_{1}^{(s, g, \alpha)}}{\left|\phi_{1}^{(s, g, \alpha)}\right|}+b^{(s, g, \alpha)} \tilde{g}(u)\right),\right.
$$




$$
\begin{aligned}
& \left.B_{|\tau| \epsilon_{0}^{(s, g, \alpha)}}\left(\frac{\tau}{\lambda_{1}^{(s, g, \alpha)}-b^{(s, g, \alpha)}} g\left(\left|\phi_{1}^{(s, g, \alpha)}\right|\right) \frac{\phi^{(s, g, \alpha)}}{\left|\phi_{1}^{(s, g, \alpha)}\right|}\right), 0\right) \\
= & (-1) \times(-1)=+1
\end{aligned}
$$

since $u=g^{-1}\left(\frac{\tau}{\lambda_{1}^{(s, g, \alpha)}-b^{(s, g, \alpha)}} g\left(\left|\phi_{1}^{(s, g, \alpha)}\right|\right) \frac{\phi^{(s, g, \alpha)}}{\left|\phi_{1}^{(s, g, \alpha)}\right|}\right)$ is the unique solution of (1.1) with $B_{|\tau| \epsilon_{0}^{(s, g, \alpha)}}\left(\frac{\tau}{\lambda_{1}^{(s, g, \alpha)}-b^{(s, g, \alpha)}} g\left(\left|\phi_{1}^{(s, g, \alpha)}\right|\right) \frac{\phi^{(s, g, \alpha)}}{\left|\phi_{1}^{(s, g, \alpha)}\right|}\right)$ and since there are two eigenvalues $\lambda_{1}^{(s, g, \alpha)}, \lambda_{2}^{(s, g, \alpha)}$ of $(-\Delta)_{g}^{s}$ to the left of $b^{(s, g, \alpha)}$ and thus the operator $\tilde{g}-\left(\tilde{g} \circ\left((-\Delta)_{g}^{s}\right)^{-1}\right) b^{(s, g, \alpha)} \tilde{g}$ has two negative eigenvalues, while all the rest are positive. When $\lambda^{(s, g, \alpha)}=1$, we have

$$
\begin{aligned}
& d_{L S}\left(\tilde{g}(u)-\left(\tilde{g} \circ\left((-\Delta)_{g}^{s}\right)^{-1}\right)\left(\tau g\left(\left|\phi_{1}^{(s, g, \alpha)}\right|\right) \frac{\phi_{1}^{(s, g, \alpha 0}}{\left|\phi_{1}^{(s, g, \alpha)}\right|}+b^{(s, g, \alpha)} \tilde{g}(u)\right.\right. \\
& +1\left(b^{(s, g, \alpha)} \tilde{g}(u)^{+}-a^{(s, g, \alpha)} \tilde{g}(u)^{-}-b^{(s, g, \alpha)} \tilde{g}(u)\right), \\
& \left.B_{|\tau| \epsilon_{0}^{(s, g, \alpha)}}\left(\frac{\tau}{\lambda_{1}^{(s, g, \alpha)}-b^{(s, g, \alpha)}} g\left(\left|\phi_{1}^{(s, g, \alpha)}\right|\right) \frac{\phi^{(s, g, \alpha)}}{\left|\phi_{1}^{(s, g, \alpha)}\right|}\right), 0\right) \\
& =d_{L S}\left(\tilde{g}(u)-\left(\tilde{g} \circ\left((-\Delta)_{g}^{s}\right)^{-1}\right)\left(\tau g\left(\left|\phi_{1}^{(s, g, \alpha)}\right|\right) \frac{\phi_{1}^{(s, g, \alpha 0}}{\left|\phi_{1}^{(s, g, \alpha)}\right|}+b^{(s, g, \alpha)} \tilde{g}(u)^{+}-a^{(s, g, \alpha)} \tilde{g}(u)^{-}\right)\right. \text {, } \\
& \left.B_{|\tau| \epsilon_{0}^{(s, g, \alpha)}}\left(\frac{\tau}{\lambda_{1}^{(s, g, \alpha)}-b^{(s, g, \alpha)}} g\left(\left|\phi_{1}^{(s, g, \alpha)}\right|\right) \frac{\phi^{(s, g, \alpha)}}{\left|\phi_{1}^{(s, g, \alpha)}\right|}\right), 0\right) \text {. }
\end{aligned}
$$

By the homotopy invariance of degree, we have

$$
\begin{gathered}
d_{L S}\left(\tilde{g}(u)-\left(\tilde{g} \circ\left((-\Delta)_{g}^{s}\right)^{-1}\right)\left(\tau g\left(\left|\phi_{1}^{(s, g, \alpha)}\right|\right) \frac{\phi_{1}^{(s, g, \alpha 0}}{\left|\phi_{1}^{(s, g, \alpha)}\right|}+b^{(s, g, \alpha)} \tilde{g}(u)^{+}-a^{(s, g, \alpha)} \tilde{g}(u)^{-}\right),\right. \\
\left.B_{|\tau| \epsilon_{0}^{(s, g, \alpha)}}\left(\frac{\tau}{\lambda_{1}^{(s, g, \alpha)}-b^{(s, g, \alpha)}} g\left(\left|\phi_{1}^{(s, g, \alpha)}\right|\right) \frac{\phi^{(s, g, \alpha)}}{\left|\phi_{1}^{(s, g, \alpha)}\right|}\right), 0\right) \\
=d_{L S}\left(\tilde{g}(u)-\left(\tilde{g} \circ\left((-\Delta)_{g}^{s}\right)^{-1}\right)\left(\tau g\left(\left|\phi_{1}^{(s, g, \alpha)}\right|\right) \frac{\phi_{1}^{(s, g, \alpha 0}}{\left|\phi_{1}^{(s, g, \alpha)}\right|}+b^{(s, g, \alpha)} \tilde{g}(u)\right),\right. \\
\left.B_{|\tau| \epsilon_{0}^{(s, g, \alpha)}}\left(\frac{\tau}{\lambda_{1}^{(s, g, \alpha)}-b^{(s, g, \alpha)}} g\left(\left|\phi_{1}^{(s, g, \alpha)}\right|\right) \frac{\phi^{(s, g, \alpha)}}{\left|\phi_{1}^{(s, g, \alpha)}\right|}\right), 0\right)=1 .
\end{gathered}
$$

Thus, the lemma is proved.

Lemma 4.5 Assume that $-\infty<a^{(s, g, \alpha)}<\lambda_{1}^{(s, g, \alpha)}, \lambda_{2}^{(s, g, \alpha)}<b^{(s, g, \alpha)}<\lambda_{3}^{(s, g, \alpha)}$ and $\tau_{1}^{(s, g, \phi)}<0$. Then, there exist a constant $\epsilon_{1}^{(s, g, \alpha)}>0$ depending on $a^{(s, g, \alpha)}, b^{(s, g, \alpha)}, \tau$ and $\tau_{1}^{(s, g, \alpha)}$ such that for any $\tau$ such that $\tau_{1}^{(s, g, \alpha)} \leq \tau<0$, the Leray-Schauder degree

$$
\begin{aligned}
& d_{L S}\left(\tilde{g}(u)-\left(\tilde{g} \circ\left((-\Delta)_{g}^{s}\right)^{-1}\right)\left(b^{(s, g, \alpha)} g(|u|) \frac{u^{+}}{|u|}-a^{(s, g, \alpha)} g(|u|) \frac{u^{-}}{|u|}+\tau g\left(\phi_{1}^{(s, g, \alpha)}\right) \frac{\phi_{1}^{(s, g, \alpha)}}{\left|\phi_{1}^{(s, g, \alpha)}\right|}\right)\right. \\
& \left.B_{|\tau| \epsilon_{1}^{(s, g, \alpha)}}\left(\tilde{g}\left(u_{2}\right)\right), 0\right)=1,
\end{aligned}
$$


where $u_{2}=-g^{-1}\left(\frac{\tau}{a^{(s, g, \alpha)}-\lambda_{1}^{(s, g, \alpha)}} g\left(\left|\phi_{1}^{(s, g, \alpha \mid}\right|\right) \frac{\phi_{1}^{(s, g, \alpha \mid}}{\left|\phi_{1}^{(s, g, \alpha \mid}\right|}\right)<0$ is a negative solution of $(1.1)$ in $W_{0}^{s} L_{G}(\Omega) \cap C(\Omega)$.

Proof We can prove this lemma by the almost identical proof to that of Lemma 4.4.

Proof of Theorem 1.3 (iii) Let $\tau<0$. We note that there is a solution $\frac{\tau}{\lambda_{1}^{(s, g, \alpha)}-b^{(s, g, \alpha)}} \times$ $g\left(\left|\phi_{1}^{(s, g, \alpha)}\right|\right) \frac{\phi_{1}^{(s, g, \alpha)}}{\left|\phi_{1}^{(s, g, \alpha)}\right|}>0 \quad$ in $\quad B_{|\tau| \epsilon_{0}^{(s, g, \alpha)}}\left(\frac{\tau}{\lambda_{1}^{(s, g, \alpha)}-b^{(s, g, \alpha)}} g\left(\left|\phi_{1}^{(s, g, \alpha)}\right|\right) \frac{\phi_{1}^{(s, g, \alpha)}}{\left|\phi_{1}^{(s, g, \alpha)}\right|}\right) \quad$ and $\quad$ a solution $\tilde{g}\left(-g^{-1}\left(\frac{\tau}{a^{(s, g, \alpha)}-\lambda_{1}^{(s, g, \alpha)}} g\left(\left|\phi_{1}^{(s, g, \alpha)}\right|\right) \frac{\phi_{1}^{(s, g, \alpha)}}{\left|\phi_{1}^{(s, g, \alpha)}\right|}\right)\right)<0 \quad$ in $\quad B_{|\tau| \epsilon_{1}^{(s, g, \alpha)}}\left(\tilde{g}\left(-g^{-1}\left(\frac{\tau}{a^{(s, g, \alpha)}-\lambda_{1}^{(s, g, \alpha)}} g\left(\left|\phi_{1}^{(s, g, \alpha)}\right|\right) \times\right.\right.\right.$ $\left.\left.\frac{\phi_{1}^{(s, g, \alpha)}}{\left|\phi_{1}^{(s, g, \alpha)}\right|}\right)\right)$ ). Let us choose $\epsilon_{0}^{(s, g, \alpha)}>0$ and $\epsilon_{1}^{(s, g, \alpha)}>0$ such that $\max \left\{\epsilon_{0}^{(s, g, \alpha)}, \epsilon_{1}^{(s, g, \alpha)}\right\}<$ $\min \left\{\frac{1}{\left|\lambda_{1}^{(s, g, \alpha)}-b^{(s, g, \alpha)}\right|}, \frac{1}{\left|a^{(s, g, \alpha)}-\lambda_{1}^{(s, g, \alpha)}\right|}\right\}$. Then, these two balls $B_{|\tau| \epsilon_{0}^{(s, g, \alpha)}}\left(\frac{\tau}{\lambda_{1}^{(s, g, \alpha)}-b^{(s, g, \alpha)}} g\left(\left|\phi_{1}^{(s, g, \alpha)}\right|\right) \times\right.$ $\left.\frac{\phi_{1}^{(s, g, \alpha)}}{\left|\phi_{1}^{(s, g, \alpha)}\right|}\right)$ and $B_{|\tau| \epsilon_{1}^{(s, g, \alpha)}}\left(\tilde{g}\left(-g^{-1}\left(\frac{\tau}{a^{(s, g, \alpha)}-\lambda_{1}^{(s, g, \alpha)}} g\left(\left|\phi_{1}^{(s, g, \alpha)}\right|\right) \frac{\phi_{1}^{(s, g, \alpha)}}{\left|\phi_{1}^{(s, g, \alpha)}\right|}\right)\right)\right.$ are disjoint. This gives two solutions. There is a large ball $B_{R^{(s, g, \alpha)}}(0)$ centred at the origin and containing

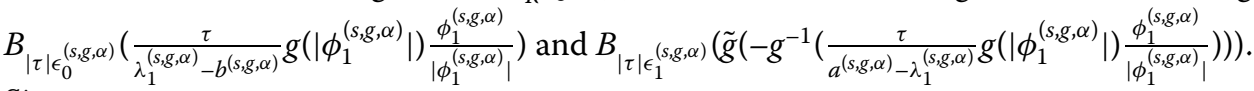

Since

$$
\begin{aligned}
& d_{L S}\left(\tilde{g}(u)-\left(\tilde{g} \circ\left((-\Delta)_{g}^{s}\right)^{-1}\right)\left(b^{(s, g, \alpha)} g(|u|) \frac{u^{+}}{|u|}-a^{(s, g, \alpha)} g(|u|) \frac{u^{-}}{|u|}+\tau g\left(\left|\phi_{1}^{(s, g, \alpha)}\right|\right) \frac{\phi_{1}^{(s, g, \alpha)}}{\left|\phi_{1}^{s, g, \alpha}\right|}\right)\right. \\
& \left.B_{R^{(s, g, \alpha)}}(0), 0\right)=0
\end{aligned}
$$

and

$$
\begin{aligned}
& d_{L S}\left(\tilde{g}(u)-\left(\tilde{g} \circ\left((-\Delta)_{g}^{s}\right)^{-1}\right)\left(b^{(s, g, \alpha)} g(|u|) \frac{u^{+}}{|u|}-a^{(s, g, \alpha)} g(|u|) \frac{u^{-}}{|u|}\right.\right. \\
& \left.\left.\quad+\tau g\left(\left|\phi_{1}^{(s, g, \alpha)}\right|\right) \frac{\phi_{1}^{(s, g, \alpha)}}{\left|\phi_{1}^{(s, g, \alpha)}\right|}\right), B_{|\tau| \epsilon_{0}^{(s, g, \alpha)}}\left(\frac{\tau}{\lambda_{1}^{(s, g, \alpha)}-b^{(s, g, \alpha)}} g\left(\left|\phi_{1}^{(s, g, \alpha)}\right|\right)\right), 0\right) \\
& =d_{L S}\left(\tilde{g}(u)-\left(\tilde{g} \circ\left((-\Delta)_{g}^{s}\right)^{-1}\right)\left(b^{(s, g, \alpha)} g(|u|) \frac{u^{+}}{|u|}-a^{(s, g, \alpha)} g(|u|) \frac{u^{-}}{|u|}\right.\right. \\
& \left.\left.\left.+\tau g\left(\phi_{1}^{(s, g, \alpha)}\right) \frac{\phi_{1}^{(s, g, \alpha)}}{\left|\phi_{1}^{(s, g, \alpha)}\right|}\right), \quad \tilde{\tau}\left(-g^{-1}\left(\frac{\tau}{a^{(s, g, \alpha)}-\lambda_{1}^{(s, g, \alpha)}} g\left(\left|\phi_{1}^{(s, g, \alpha \mid}\right|\right) \frac{\phi_{1}^{(s, g, \alpha \mid}}{\left|\phi_{1}^{(s, g, \alpha \mid}\right|}\right)\right)\right), 0\right)=1,
\end{aligned}
$$

it follows that

$$
\begin{gathered}
d_{L S}\left(\tilde{g}(u)-\left(\tilde{g} \circ\left((-\Delta)_{g}^{s}\right)^{-1}\right)\left(b^{(s, g, \alpha)} g(|u|) \frac{u^{+}}{|u|}-a^{(s, g, \alpha)} g(|u|) \frac{u^{-}}{|u|}+\tau g\left(\phi_{1}\right) \frac{\phi_{1}^{(s, g, \alpha)}}{\left|\phi_{1}^{s, g, \alpha}\right|}\right),\right. \\
B_{R^{(s, g, \alpha)}}(0) \backslash\left(B_{|\tau| \epsilon_{0}^{(s, g, \alpha)}}\left(\frac{\tau}{\lambda_{1}^{(s, g, \alpha)}-b^{(s, g, \alpha)}} g\left(\left|\phi_{1}^{(s, g, \alpha)}\right|\right) \frac{\phi_{1}^{(s, g, \alpha)}}{\left|\phi_{1}^{s, g, \alpha}\right|}\right)\right. \\
\left.\left.\cup B_{|\tau| \epsilon_{1}^{(s, g, \alpha)}}\left(\tilde{g}\left(-g^{-1}\left(\frac{\tau}{a^{(s, g, \alpha)}-\lambda_{1}^{(s, g, \alpha)}} g\left(\left|\phi_{1}^{(s, g, \alpha \mid}\right|\right) \frac{\phi_{1}^{(s, g, \alpha \mid}}{\left|\phi_{1}^{(s, g, \alpha \mid}\right|}\right)\right)\right)\right), 0\right)=-2 .
\end{gathered}
$$


Thus, there exists a third solution $\tilde{g}(u)$ in $B_{R^{(s, g, \alpha)}}(0) \backslash\left(B_{|\tau| \epsilon \epsilon_{0}^{(s, g, \alpha)}}\left(\frac{\tau}{\lambda_{1}^{(s, g, \alpha)}-b^{(s, g, \alpha)}} g\left(\left|\phi_{1}^{(s, g, \alpha)}\right|\right)\right) \cup\right.$ $B_{|\tau| \epsilon_{1}^{(s, g, \alpha)}}\left(\tilde{g}\left(-g^{-1}\left(\frac{\tau}{a^{(s, g, \alpha)}-\lambda_{1}^{(s, g, \alpha)}} g\left(\left|\phi_{1}^{(s, g, \alpha \mid}\right|\right) \frac{\phi_{1}^{(s, g, \alpha \mid}}{\left|\phi_{1}^{(s, g, \alpha \mid}\right|}\right)\right)\right)$ such that $u$ is a solution of $(1.1)$ in $W_{0}^{s} L_{G}(\Omega) \cap$ $C(\Omega)$. Thus, there exists a third solution $u$ of $(1.1)$ in $W_{0}^{s} L_{G}(\Omega) \cap C(\Omega)$. Thus, we prove (iii) of Theorem 1.3.

\section{Acknowledgements}

Not applicable

\section{Funding}

Q-Heung Choi was supported by the Basic Science Research Program through the National Research Foundation of Korea (NRF) funded by the Ministry of Education, Science and Technology (NRF-2017R1D1A1B03030024). Tacksun Jung was supported by the Basic Science Research Program through the National Research Foundation of Korea (NRF) funded by the Ministry of Science, ICT and Future Planning (NRF-2017R1A2B4005883).

\section{Abbreviations}

Not applicable.

\section{Availability of data and materials}

Not applicable

\section{Declarations}

\section{Competing interests}

The authors declare that there is no conflict of interests regarding the publication of this paper

\section{Authors' contributions}

Q-HC introduced the main ideas of multiplicity study for this problem. TJ participated in applying the method for solving this problem and drafted the manuscript. All authors contributed equally to, read and approved the final manuscript.

\section{Author details}

'Department of Mathematics Education, Inha University, Incheon 402-751, Korea. ${ }^{2}$ Department of Mathematics, Kunsan National University, Kunsan, 573-701, Korea.

\section{Publisher's Note}

Springer Nature remains neutral with regard to jurisdictional claims in published maps and institutional affiliations.

Received: 30 June 2021 Accepted: 12 November 2021 Published online: 04 December 2021

\section{References}

1. Alberico, A., Cianchi, A., Pick, L., Slavíková, L.: Fractional Orlicz-Sobolev embeddings. Journal de Mathématiques Pures et Appliquées 149(5), 216-253 (2021)

2. Applebaum, D.: Lévy processes-from probability to finance and quantum groups. Not. Am. Math. Soc. 51(2), 1336-1347 (2004)

3. Azroul, E., Benkirane, A., Srati, M.: Existence of solutions for a nonlocal type problem in fractional Orlicz-Sobolev spaces (2019). preprint

4. Bahrouni, S., Ounaies, H.: Embedding theorems in the fractional Orlicz-Sobolev space and applications to non-local problems. Discrete Contin. Dyn. Syst. 40(5), 2917-2944 (2020)

5. Bahrouni, S., Ounaies, H., Salort, A.M.: Variational eigenvalues of the fractional $g$-Laplacian. 2011.14742

6. Bahrouni, S., Ounaies, H., Tavares, L.S.: Basic results of fractional Orlicz-Sobolev space and applications to non-local problems. Topol. Methods Nonlinear Anal. 55(2), 681-695 (2020)

7. Bonder, J.F., Salort, A.M.: Fractional order Orlicz-Sobolev spaces. J. Funct. Anal. 277(2), 333-367 (2019)

8. Choi, Q.H., Jung, T.: An application of a variational reduction method to a nonlinear wave equation. J. Differ. Equ. 117, 390-410 (1995)

9. Choi, Q.H., Jung, T.: A nonlinear suspension bridge equation with nonconstant load. Nonlinear Anal. TMA 35, 649-668 (1999)

10. Choi, Q.H., Jung, T.: Multiplicity results for the nonlinear suspension bridge equation. Dyn. Contin. Discrete Impuls. Syst., Ser. A Math. Anal. 9, 29-38 (2002)

11. Choi, Q.H., Jung, T., McKenna, P.J.: The study of a nonlinear suspension bridge equation by a variational reduction method. Appl. Anal. 50, 73-92 (1993)

12. Cont, R., Tankov, P.: Financial Modelling with Jump Processes. Financial Mathematics Series, vol. 2. Chapman and Hall/CRC, Boca Raton (2004)

13. Garciá-Huidobro, M., Le, V.K., Manásevich, R., Schmitt, K.: On principle eigenvalues for quasilinear elliptic differential operators: an Orlicz-Sobolev space setting. NoDEA Nonlinear Differ. Equ. Appl. 6, 207-225 (1999)

14. Kaufmann, U., Rossi, J.D., Vidal, R.: Fractional Sobolev spaces with variable exponents and $p(x)$-Laplacians. Electron. J. Qual. Theory Differ. Equ. 76, 1 (2017) 
15. McKenna, P.J., Walter, W.: Nonlinear oscillations in a suspension bridge. Arch. Ration. Mech. Anal. 98, 167-177 (1987)

16. Mihăilescu, M., Rădulescu, V.: Neumann problems associated to nonhomogeneous differential operators in Orlicz-Sobolev spaces. Ann. Inst. Fourier 58, 2087-2111 (2008)

17. Osanchol, A.: A note on the Definition of an Orlicz Space. https://doi.org/10.5578/fmbd.8672

18. Rao, M.M., Ren, Z.D.: Theory of Orlicz Spaces. Dekker, New York (1991)

19. Rúžička, M.: Electrorheological Fluids: Modeling and Mathematical Theory. Lecture Notes in Mathematics, vol. 1748. Springer, Berlin (2000)

20. Salort, A.M.: Eigenvalues and minimizers for a non-standard growth non-local operator. J. Differ. Equ. 268(9), 5413-5439 (2020)

21. Salort, A.M.: Hardy inequalities in fractional Orlicz-Sobolev spaces (14 Sep 2020) 2009.06431v1 [math.AP]

\section{Submit your manuscript to a SpringerOpen ${ }^{\circ}$} journal and benefit from:

- Convenient online submission

- Rigorous peer review

- Open access: articles freely available online

- High visibility within the field

- Retaining the copyright to your article

Submit your next manuscript at $\boldsymbol{\Delta}$ springeropen.com 